ARTICLE

\title{
Mitochondrial function provides instructive signals for activation-induced B-cell fates
}

\author{
Kyoung-Jin Jang ${ }^{1}$, Hiroto Mano ${ }^{1}$, Koji Aoki ${ }^{2}$, Tatsunari Hayashi ${ }^{1}$, Akihiko Muto ${ }^{3,4}$, Yukiko Nambu ${ }^{1, \dagger}$, \\ Katsu Takahashi ${ }^{5}$, Katsuhiko Itoh $^{6}$, Shigeru Taketani ${ }^{7}$, Stephen L. Nutt ${ }^{8,9}$, Kazuhiko Igarashi ${ }^{3,4}$, \\ Akira Shimizu ${ }^{1} \&$ Manabu Sugai ${ }^{1,10}$
}

During immune reactions, functionally distinct B-cell subsets are generated by stochastic processes, including class-switch recombination (CSR) and plasma cell differentiation (PCD). In this study, we show a strong association between individual B-cell fates and mitochondrial functions. CSR occurs specifically in activated $B$ cells with increased mitochondrial mass and membrane potential, which augment mitochondrial reactive oxygen species (mROS), whereas PCD occurs in cells with decreased mitochondrial mass and potential. These events are consequences of initial slight changes in $\mathrm{mROS}$ in mitochondria high $\mathrm{B}$-cell populations. In CSR-committed cells, mROS attenuates haeme synthesis by inhibiting ferrous ion addition to protoporphyrin IX, thereby maintaining Bach2 function. Reduced mROS then promotes PCD by increasing haeme synthesis. In PCD-committed cells, Blimp1 reduces mitochondrial mass, thereby reducing mROS levels. Identifying $\mathrm{mROS}$ as a haeme synthesis regulator increases the understanding of mechanisms regulating haeme homeostasis and cell fate determination after B-cell activation.

\footnotetext{
${ }^{1}$ Department of Experimental Therapeutics, Institute for Advancement of Clinical and Translational Science, Kyoto University Hospital, 54 Shogoin-Kawahara-cho, Sakyo-ku, Kyoto 606-8507, Japan. ${ }^{2}$ Division of Pharmacology, School of Medicine, University of Fukui, 23-3 Matsuokashimoaizuki, Eiheiji-cho, Yoshida-gun, Fukui 910-1193, Japan. ${ }^{3}$ Department of Biochemistry, Tohoku University Graduate School of Medicine, Seiryo-machi 2-1, Aoba-ku, Sendai, Miyagi 980-8575, Japan. ${ }^{4}$ CREST, Japan Science and Technology Agency, Seiryo-machi 2-1, Aoba-ku, Sendai, Miyagi 980-8575, Japan. ${ }^{5}$ Department of Oral and Maxillofacial Surgery, Kyoto University Hospital, 54 Shogoin-Kawahara-cho, Sakyo-ku, Kyoto 606-8507, Japan. ${ }^{6}$ Department of Clinical Molecular Biology, Graduate School of Medicine, Kyoto University, 54 Shogoin-Kawahara-cho, Sakyo-ku, Kyoto 606-8507, Japan. ${ }^{7}$ Department of Biotechnology, Kyoto Institute of Technology, Matsugasaki, Sakyo-ku, Kyoto 606-8585, Japan. ${ }^{8}$ The Walter and Eliza Hall Institute of Medical Research, Melbourne, Victoria 3052, Australia. ${ }^{9}$ Department of Medical Biology, University of Melbourne, Victoria 3010, Australia. ${ }^{10}$ Division of Molecular Genetics, Department of Biochemistry and Bioinformative Sciences, School of Medicine, University of Fukui, 23-3 Matsuokashimoaizuki, Eiheiji-cho, Yoshida-gun, Fukui 910-1193, Japan. † Present address: Bioprocessing Technology Institute, A*STAR20 Biopolis Way \#06-01 Centros, Singapore 138668, Singapore. Correspondence and requests for materials should be addressed to M.S. (email: msugai@u-fukui.ac.jp).
} 
$\mathrm{n}$ antigen challenge, naïve $\mathrm{B}$ lymphocytes undergo diversification of their antigen receptor via somatic hypermutation (SHM), alteration of immunoglobulin function by class-switch recombination $(\mathrm{CSR})^{1-7}$ and differentiation into antibody-secreting plasma cells or memory $\mathrm{B}$ cells $^{8-13}$. Although several important transcription factors involved in these processes have been identified, the interrelations in the regulatory network that determine cell fates after B-cell activation remain elusive ${ }^{14-17}$. Pax5 and Bach2 are required for CSR because ablations of these genes in $\mathrm{B}$ cells destroy the ability of the cell to undergo $\mathrm{CSR}^{2,18}$. Pax5 and Bach2 also inhibit plasma cell differentiation (PCD) by inhibiting the transcription of Blimp1, a master gene for PCD ${ }^{2,19-22}$. Irf4 and Xbp1 act in concert with Blimp1 to establish plasma cell fate ${ }^{8,11,13,23,24}$. However, Blimp1 represses Pax5 expression in differentiating plasma cells ${ }^{9,10}$. The transcription factor Irf4 plays a pivotal regulatory role in these processes because it is required for affinity maturation, CSR and PCD ${ }^{11-13}$. An intermediate amount of Irf4 induces the expression of Bcl6 and activation-induced cytidine deaminase (AID), whereas a high amount induces the expression of Blimp1 (refs 12,13). CSR and SHM are initiated by the AIDdependent cytidine deamination of DNA in immunoglobulin loci ${ }^{1,3-6}$. Bcl6 is required for germinal-centre (GC) B-cell development. During the GC reaction, B cells undergo SHM at their immunoglobulin loci, and high-affinity immunoglobulinbearing $\mathrm{B}$ cells are selected in a process called affinity maturation ${ }^{25-29}$. Mutually exclusive expression of Bcl6 and Blimp1 is established by the mutual inhibition of expression. Although the essential gene sets for various B-cell fates after activation and a regulatory network that inhibits unselected fates have been described, the instructive signals for the initiation of the respective B-cell fates or transitions between them are not completely known.

Metabolism encompasses the generation of energy via catabolism and of macromolecules via anabolism. Recent studies have indicated more complex roles of metabolic pathways or metabolites in cell differentiation and function ${ }^{30-33}$. A shift towards glycolysis occurs in macrophages activated by lipopolysaccharide (LPS), inducing interleukin- $1 \beta$ production in a hypoxia-inducible factor 1 -alpha-dependent manner. Inhibition of glycolysis using 2-deoxyglucose suppresses interleukin-1 $\beta$ production via the reduction of succinate, an intermediate of the tricarboxylic acid (TCA) cycle or the $\gamma$-aminobutyric acid shunt, and stabilizes HIF-1 $\alpha$ (ref. 31). A strong bias towards glycolysis over mitochondrial metabolism has also been observed in haematopoietic stem cell (HSC) maintenance ${ }^{34}$, Th17 differentiation, tumour cell functions and interferon- $\gamma$ production in activated $\mathrm{T}$ cells $\mathrm{s}^{30}$. However, regulatory $\mathrm{T}$ cells display a mixed metabolism that includes lipid oxidation, oxidative phosphorylation and glycolysis ${ }^{30}$. In addition to generating various metabolites in mitochondria, mitochondriaderived reactive oxygen species (mROS) are now understood to function as signalling molecules ${ }^{35}$. This indicates that mitochondria play fundamental roles in cell differentiation and function. In activated B cells, haeme strongly induces PCD by inhibiting Bach2 function ${ }^{36}$.

Because activated B cells differentiate into various cell types in a stochastic manner ${ }^{15}$, we speculated that stochastic changes in some metabolic pathways would provide instructive signals to activated B cells. In this study, we investigated the functions of mitochondria in fate determination of activated B cells and attempted to identify the signals directing B-cell fate towards CSR or PCD. We first observed that differences in mitochondrial status predicted the direction of the committed cells toward either pathway. CSR occurred in activated B cells with increased mitochondrial mass and membrane potential, whereas PCD occurred in cells with decreased mitochondrial mass and potential. However, differences in mitochondrial mass and membrane potential were the consequences of slight initial changes in generating mROS within the mitochondriahigh $\mathrm{B}$-cell population. Activated mitochondriahigh $B$ cells with higher amounts of mROS maintained increased mitochondrial mass and membrane potential, whereas cells with lower amounts displayed decreased mitochondrial mass and potential. Thus, slight differences in mROS were the initial signal in cell fate determination after B-cell activation. Notably, the choice for the B-cell fate of PCD or CSR was dependent on mitochondrial functional activity that regulated an intracellular ROS level, which suppressed haeme synthesis. These results suggest that mitochondria contribute to B-cell fate determination via the integration of instructive and cell-intrinsic stochastic signals by regulating haeme and ROS levels.

\section{Results}

Activation-dependent generation of three B-cell populations. Because alterations of metabolic pathways or metabolites are involved in lineage commitment and cell differentiation ${ }^{31-33}$, we hypothesized that intracellular metabolism plays some role in the fate determination of the activated B cells. To test this possibility, we investigated the involvement of mitochondria in B-cell fate determination, considering that mitochondria produce various metabolites including ATP, haeme and iron-sulfur clusters. First, we evaluated total mitochondrial mass and membrane potential after B-cell activation in vitro ${ }^{37}$ using MitoTracker Green and DeepRed dyes, respectively. Mitochondrial mass and membrane potential were increased, and a relatively uniform population (called population 1, P1) was generated on day 1 of stimulation with LPS + IL-4 or anti-CD40 + IL-4 (Fig. 1a and Supplementary Fig. 1b). On day 3 of stimulation, cells showing intermediate levels of mitochondrial mass and membrane potential (population 2, P2) increased in frequency. To investigate the relationship between mitochondrial status and cell fates, we examined the mitochondrial status of class switched cells $\left(\mathrm{IgG} 1^{+}\right.$ cells) and plasma cells (CD138 ${ }^{+}$cells) on day 4 of stimulation. IgG1 ${ }^{+}$cells preferentially belonged to the $\mathrm{P} 1$ population and $\mathrm{CD} 138^{+}$cells to the P2 population (Fig. 1a and Supplementary Fig. 1a-c), suggesting an association between P1 cells and CSR and between P2 cells and PCD. Another minor population, called P3 cells, also expressed IgG1. When the same assays were performed using TMRM dye, instead of MitoTracker DeepRed, essentially the same results were obtained (Supplementary Fig. 2a). Next we evaluated total mitochondrial mass and membrane potential after B-cell activation in vivo. Again, we found P1, P2 and P3 cell populations among GC B cells during the T-cell-dependent immune reaction. P1 cells preferentially expressed IgG1, whereas P2 cells expressed CD138 (Fig. 1b). In this case, IgG1-expressing plasmablasts were also observed among P2 cells. This finding indicated that PCD occurred after IgG1 switching (Fig. 1b), because time-course analysis indicated that P2 cells were generated later than P1 cells after B-cell activation in vitro (Fig. 1a and Supplementary Fig. 1b). Same assays were performed using TMRM dye, instead of MitoTracker DeepRed, and essentially the same results were obtained (Supplementary Fig. 1d). $\mathrm{CD} 138^{+}$cells were also enriched in P2 populations within $\mathrm{GL7}^{+}$GC B cells (Supplementary Fig. 3a). We further examined mitochondrial status of splenic plasma cells in the same mice as used for Fig. 1b. Proportions of P2 populations were increased in plasma cells (Supplementary Fig. 3b). In the T-cellindependent immune response, plasma cells were also observed among P2 cells, but IgG3-expressing cells were observed among P1 cells (Supplementary Fig. 3c). Thus, there was a strong 
a
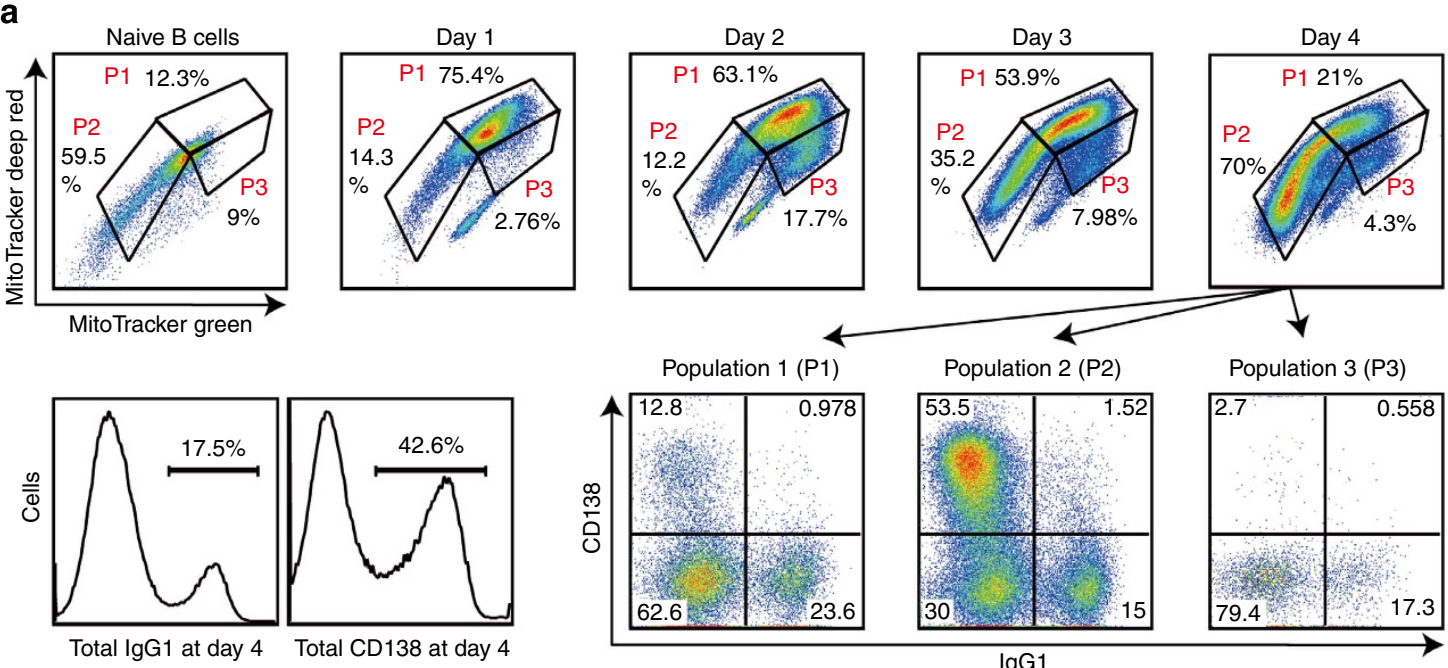

b
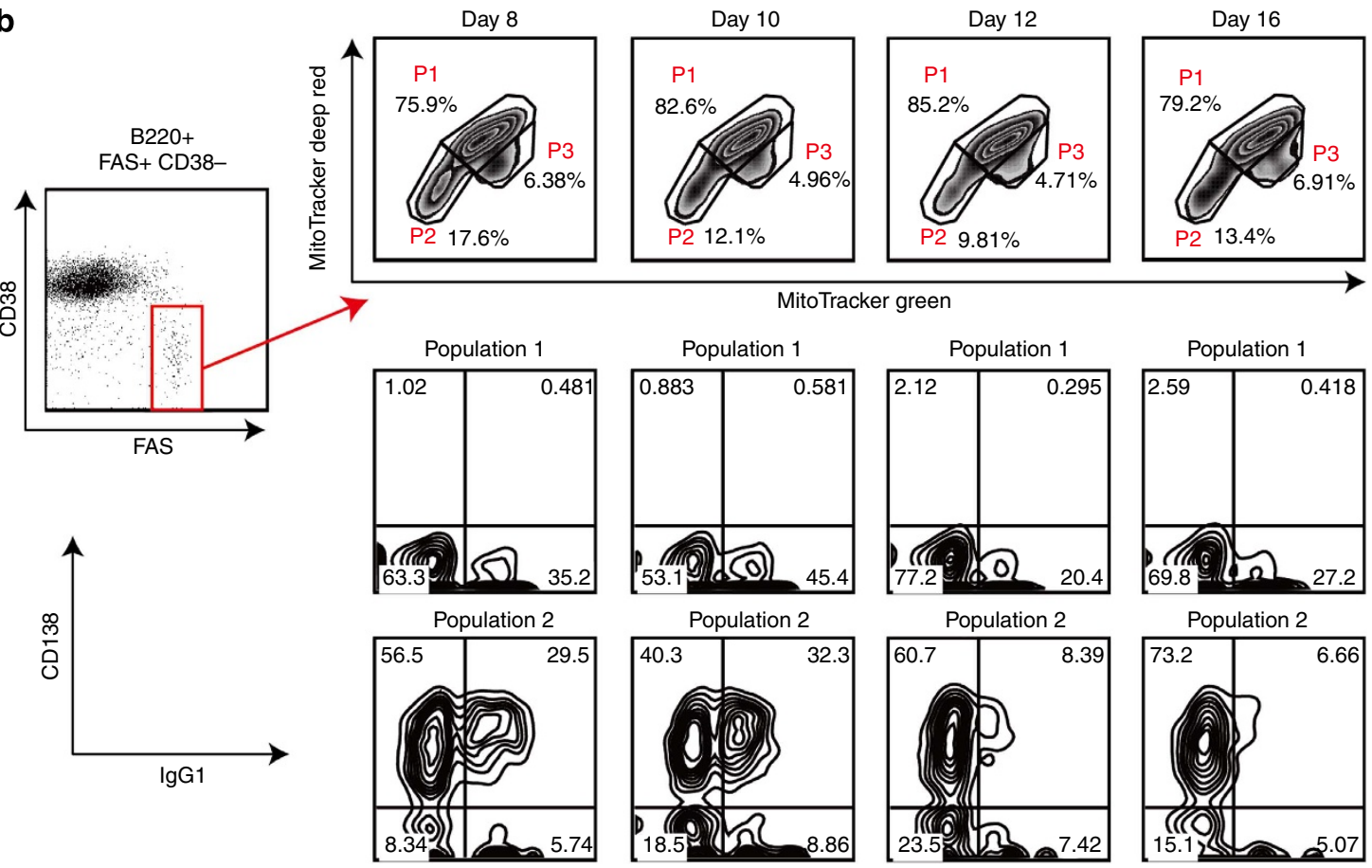

C
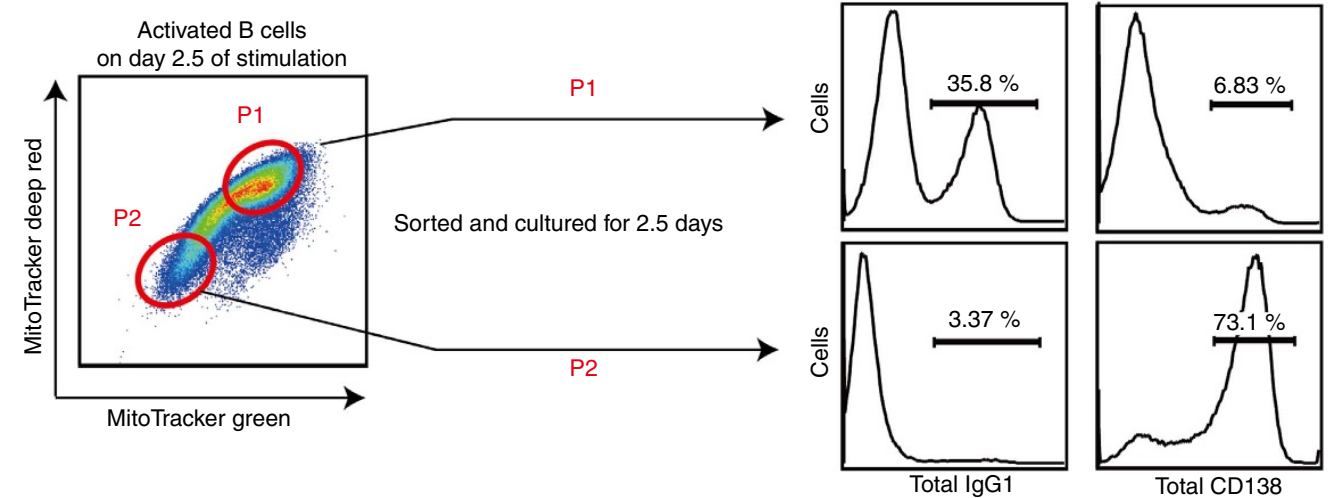

Figure 1 | Activated B cells are subdivided into three groups according to the mitochondrial status. (a) Flow cytometric analysis of mitochondrial membrane potential and size monitored by MitoTracker staining on the indicated day (top) or differentiation of the B cells monitored by CD138 and lgG1 expression on day 4 (bottom) in LPS + IL-4-stimulated B cells. (b) Flow cytometric analysis of the mitochondrial status on the indicated day after immunization (top) with NP-CGG and the differentiation status of population 1 (middle) and population 2 (bottom) in GC B cells (B220 + CD38 - FAS +). (c) Diagrammatic representation of experimental overview. Flow cytometric analysis of differentiation of sorted P1 and P2 cells. Data shown are representative of three independent experiments. 
association between mitochondrial status and B-cell fate determination. To evaluate this further, we investigated the differential abilities of differentiation of P1 and P2 cells towards CSR and PCD. To this end, we collected undifferentiated P1 and P2 cells (indicated populations in Fig. 1c) that did not express IgG1 and CD138 and stimulated them to differentiate. Consistent with the above results (Fig. 1a,b), IgG1 was expressed in more cells derived from P1 than from P2 cells (Fig. 1c), whereas CD138 was expressed in more cells derived from P2 than from P1 cells (Fig. 1c). These results suggested that undifferentiated cells found in P1 and P2 cell populations were committed to CSR and PCD, respectively.

Modulation of mitochondrial function affects B-cell fate. To investigate the contribution of mitochondrial metabolism to B-cell fate determination, we blocked key enzymes of the respiratory chain of mitochondria to reduce ATP levels. The number of cells in the P1 cell fraction was increased by the addition of the complex I inhibitors rotenone/metformin or the complex V inhibitor oligomycin, whereas PCD was strongly suppressed (Fig. 2a,b,i,j,m,n and Supplementary Fig. 4a). We also inhibited the major metabolic pathways in mitochondria to examine the involvement of distinctive catabolic pathways of glucose or fatty acids in activated B-cell fate determination. We found increases in P1 cell numbers and decreases in P2 cell numbers after treatment with 2-deoxyglucose, a glucose analogue that inhibits glycolysis, and etomoxir, an inhibitor of fatty acid oxidation (Fig. 2a,c,d and Supplementary Fig. 4a). Similarly, increased P1 cell numbers and decreased P2 cell numbers were observed after treatment with methyl pyruvate, which provides substrates for the TCA cycle, and methyl malate, which generates NADPH (Fig. 2a,e,f and Supplementary Fig. 4a). In contrast, P2 cell generation and PCD were enhanced by the addition of the antioxidant ascorbic acid, whereas CSR was suppressed (Fig. 2a,g and Supplementary Fig. 4a).

Treatment of activated $\mathrm{B}$ cells with inhibitors of the phosphatidylinositol 3-kinase (PI3K)-Akt pathway increased P1 cell numbers, enhanced CSR, reduced P2 cell numbers and suppressed PCD (Fig. 2i,k,l and Supplementary Fig. 4a). These results were consistent with earlier findings that the PI3K-Akt pathway inhibits CSR and promotes $\mathrm{PCD}^{38}$.

We further investigated the relationship between haeme and mitochondrial function in the control of B-cell fate determination because haeme is a strong inducer of PCD via the inhibition of Bach2 function ${ }^{36}$. Addition of haeme promoted skewed P2 cell generation (Fig. 2a,h and Supplementary Fig. 4a). Because CSR and PCD are influenced by cell proliferation, effects of various reagents on the number of cell divisions were assessed using carboxyfluorescein succinimidyl ester (CFSE)-labelled B cells. As shown in Supplementary Fig. $5 \mathrm{a}-\mathrm{d}, \mathrm{f}, \mathrm{h}-\mathrm{k}$, the proliferation of cells was less affected by the presence of various reagents. Methyl pyruvate inhibited cell proliferation but affected only PCD (Supplementary Fig. 5g). For etomoxir-treated cells, reduced CSR and PCD may be the results of reduced cell proliferation (Supplementary Fig. 5e). These results collectively suggested that commitment of activated B cells to CSR and PCD is controlled by mitochondrial functions, as indicated by $\mathrm{P} 1$ and $\mathrm{P} 2$ populations, irrespective of specific metabolic pathways.

Metabolic and gene expression profiles of P1 and P2 cells. To characterize cells in P1 and P2 cell populations, in vitro-activated $\mathrm{P} 1$ and P2 cells were investigated by transmission electron microscopy (Fig. 3a). Mitochondrial mass increased in P1 cells, consistent with the above results (Fig. 1). To further evaluate mitochondrial function, we determined the oxygen consumption rate (OCR) in $\mathrm{P} 1$ and $\mathrm{P} 2$ cells. Basal OCR, an indicator of oxidative phosphorylation, was 1.4 times higher in P1 than in P2 cells (Fig. 3b). The basal extracellular acidification rate (ECAR, a marker of glycolysis) was significantly higher in P1 than in P2 cells (Fig. 3b). ATP levels were consistently higher in P1 than in P2 cells (Fig. 3c). We also investigated the roles of OCR and ECAR during B-cell fate determination, using various reagents described in Fig. 2 (Supplementary Fig. 4b). However, there was no obvious involvement of OCR and ECAR in B-cell differentiation. Because mROS play key roles in various cell functions $35,39,40$, we evaluated their levels in $\mathrm{P} 1$ and $\mathrm{P} 2$ cell populations. We observed a ROS high population in $\mathrm{P} 1$ cells (Fig. 3d and Supplementary Figs $1 \mathrm{~d}$ and 2b,c). We also investigated the roles of ROS generation during B-cell fate determination using various reagents described in Fig. 2. ROS were induced by $\mathrm{P} 1$ cell-promoting reagents, whereas their levels were reduced by $\mathrm{P} 2$ cell-promoting reagents (Supplementary Figs 6-8). This finding suggested that ROS promoted CSR but suppressed PCD.

Protein levels in P1 cells were increased for Pax5, Bcl6, AID and c-Myc, which have been reported to promote CSR. Levels of Blimp1, Irf4 and Xbp1 proteins were higher in P2 than in P1 cells. The expression level of c-Myc increased in P1 cells, whereas that of Pax5 significantly decreased in P2 cells (Fig. 3e). These expression patterns were consistent with our hypothesis that P1 and P2 cells are cell populations precommitted to CSR and PCD.

Differential haeme synthesis in $\mathrm{P} 1$ and $\mathrm{P} 2$ cell populations. Because Bach2 promotes CSR and inhibits PCD via the repression of Blimp1 expression ${ }^{21,41}$, the reduced level of Bach2 correlates strongly with reciprocal Blimp1 expression in the normal course of B-cell activation. However, we found significant expression levels of both Blimp1 and Bach2 in P2 cells (Fig. 3e). Expression levels of Bach 2 and Bcl6 were similar between P1 and P2 cells. We first determined the haeme levels that inhibit Bach2 function ${ }^{36}$. Haeme levels were higher in P2 cells than in P1 cells (Fig. 4a). In vivo-generated $\mathrm{P} 2$ cells also showed higher haeme levels than P1 cells (Fig. 4a). These results indicated a direct relationship between haeme levels and mitochondrial function. We then investigated the possible role of ROS in haeme synthesis. Haeme levels (Fig. 4b,c) were increased and PCD (Figs 2 and $4 \mathrm{~d}$ and Supplementary Fig. 9) was promoted after treatment of P1 cells or activated $\mathrm{B}$ cells with ascorbic acid or MitoTEMPO, a mitochondria-targeted antioxidant. In MitoTEMPO treated cells, cellular ROS level was little reduced by day 4 (Supplementary Fig. 9c). We accordingly performed a timecourse analysis of cellular ROS and found an antioxidant effect of MitoTEMPO on day 2 cells ( $24 \mathrm{~h}$ after MitoTEMPO treatment, Supplementary Fig. 9c). These data indicated that ROS at early time points was important in B-cell fate determination.

TPP cation is usually used as a carrier for targeting antioxidant to mitochondria. Because MitoTEMPO also uses TPP, TPP is a suitable control for assessing antioxidant function of MitoTEMPO. However, TPP was toxic to activated B cells, because cell numbers of TPP-treated cells decreased (Supplementary Fig. 10a). TPP, unlike MitoTEMPO, has no specific role in CSR and PCD, as shown in Supplementary Fig. 10b. TPP inhibited CSR and PCD in a dose-dependent manner. These results collectively suggested that mROS inhibited haeme synthesis and promoted CSR.

mROS interfere with addition of ferrous ions to PpIX. The generation of 5-aminolevulinic acid (ALA) catalysed by ALA synthase is the initial step in haeme synthesis. As the final step, ferrous ion is incorporated into protoporphyrin IX (PpIX) by 
a

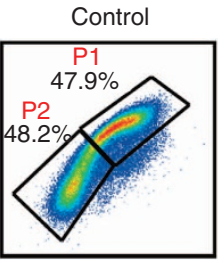

b

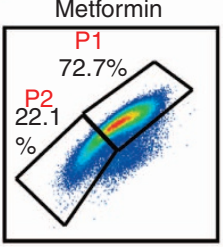

c

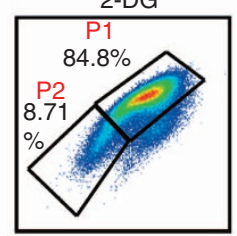

d

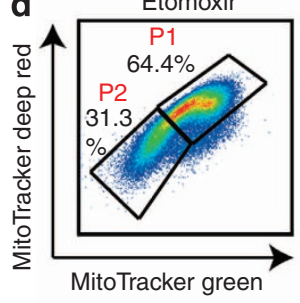

i
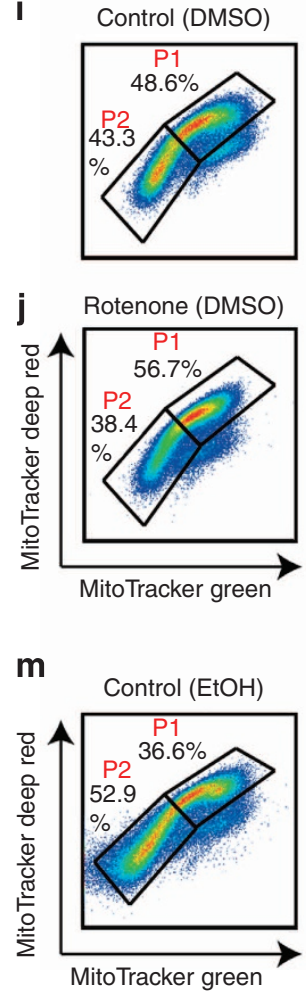
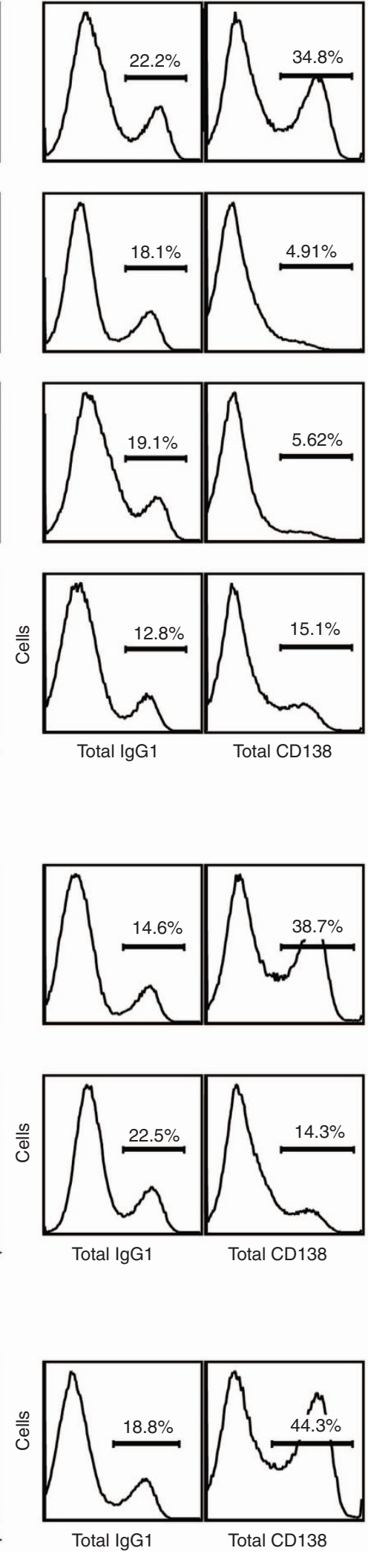

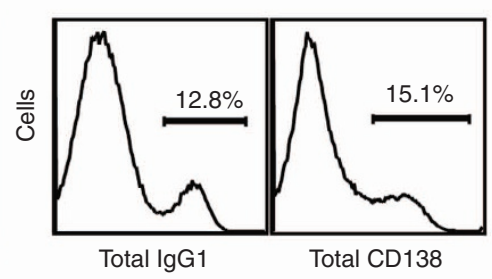

e

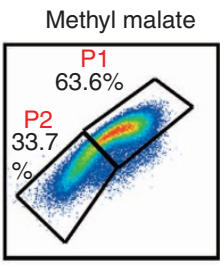

f
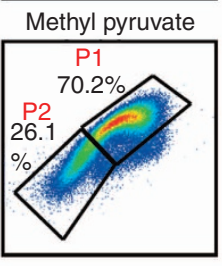

Ascorbic acid
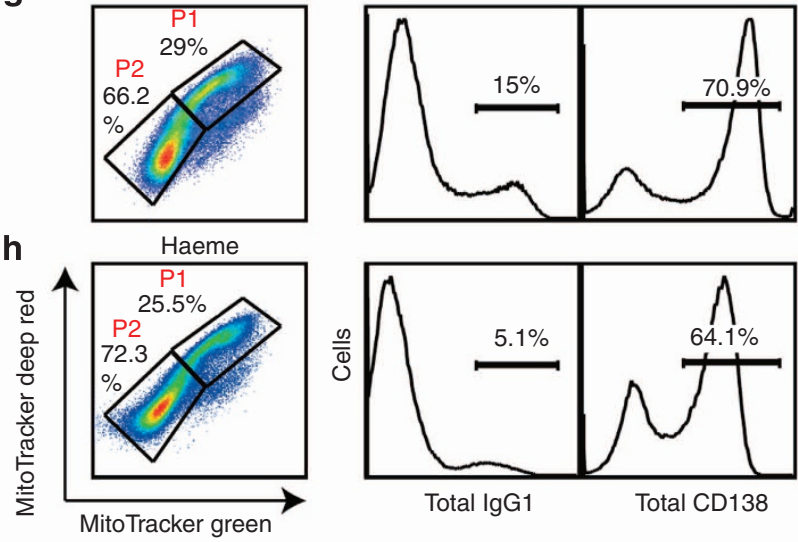

k LY294002 (DMSO) - PI3K inhibitor
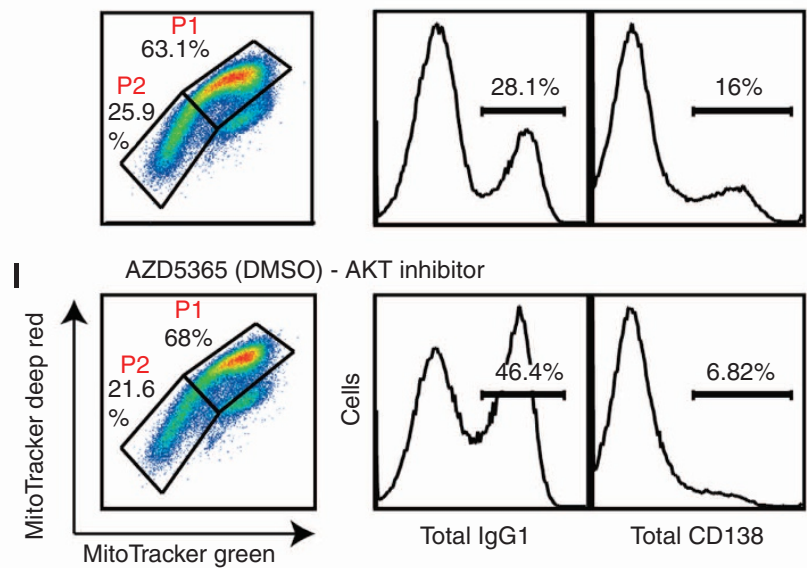

n
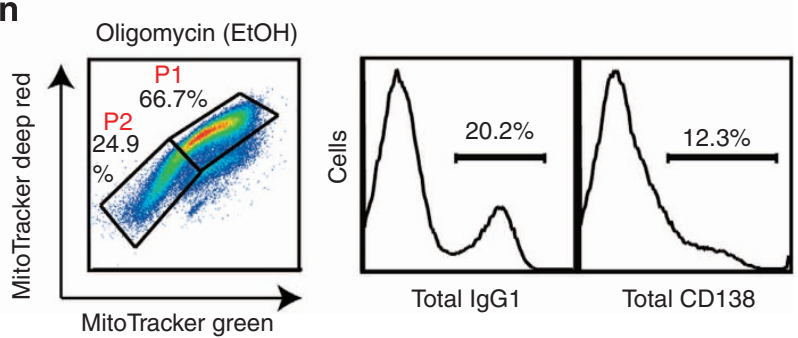

Total IgG1

Total CD138

Figure 2 | Association of mitochondrial status with activated B-cell fate. Flow cytometric analysis of mitochondrial status monitored by MitoTracker

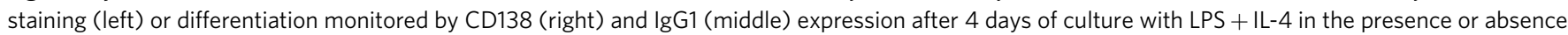
of the indicated reagents. $\mathbf{a}$ is the control for $\mathbf{b}-\mathbf{h}$. $\mathbf{i}$ is the control for $\mathbf{j}-\mathbf{I}$. $\mathbf{m}$ is the control for $\mathbf{n}$. Data shown are representative of three independent experiments. DMSO, dimethylsulphoxide. 
a

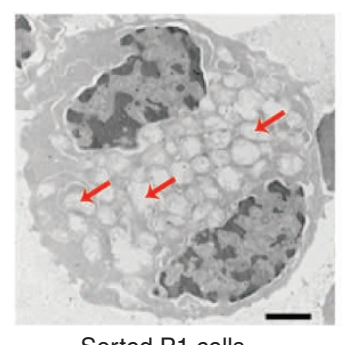

Sorted P1 cells

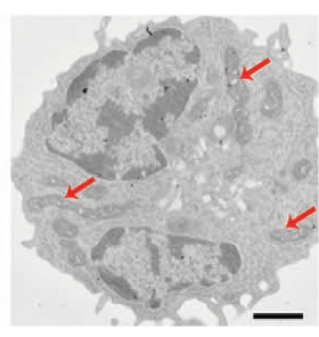

Sorted P2 cells

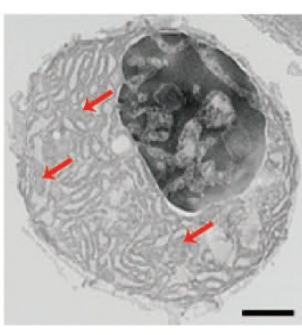

Sorted P2 cells

c

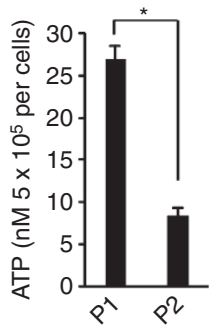

$c$
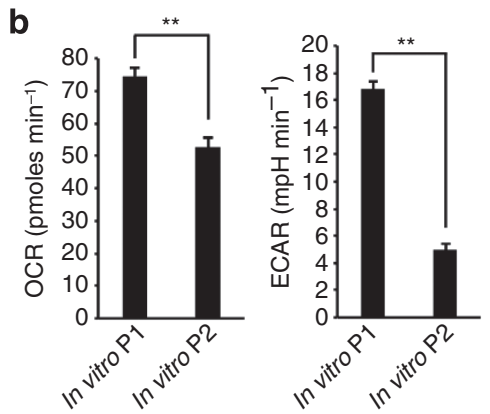

d
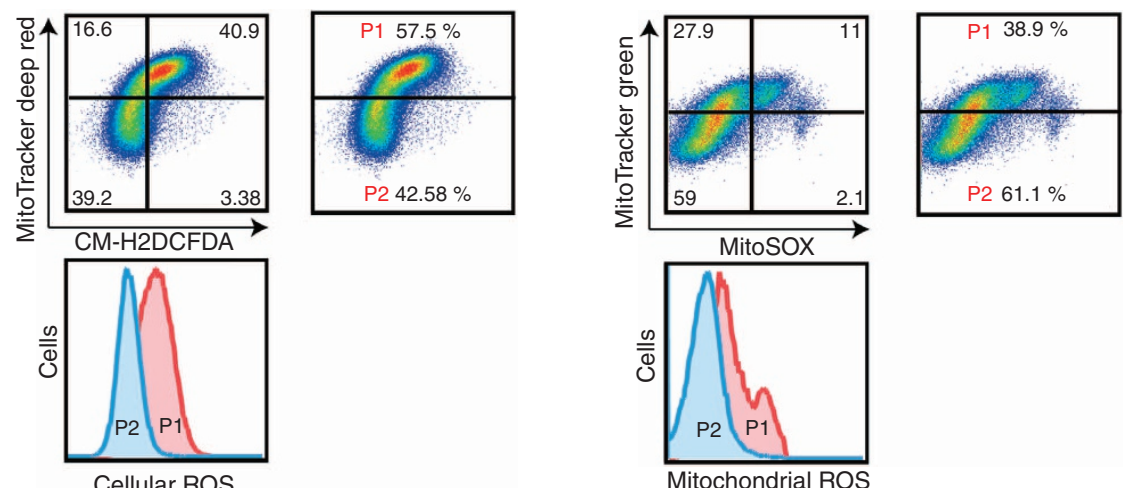

Cellular ROS

e

Activated $B$ cells

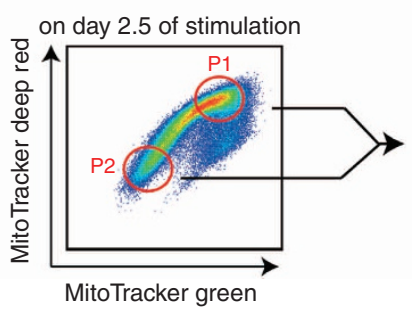

Sorted and analysed by western bolt

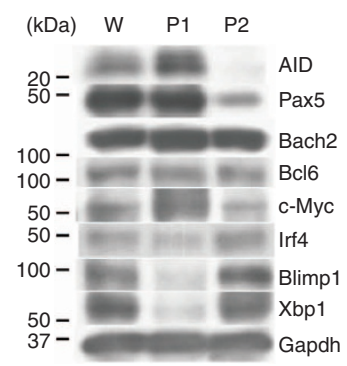

Figure 3 | Characterization of P1 and P2 cell populations. (a) Transmission electron microscopy of FACS-sorted P1 and P2 cells. Red arrow indicates mitochondria. Mitochondria in P1 cells have been damaged during the sorting procedure. A larger endoplasmic reticulum in developing P2 cells is shown (right). Scale bar, $2 \mu \mathrm{m}$. (b) OCR and ECAR of sorted P1 and P2 cells. ${ }^{\star \star} P<0.005$ (two-tailed Student's t-test). (c) ATP levels in sorted P1 and P2 cells. ${ }^{\star} P<0.05$ (two-tailed Student's t-test). (d) Flow cytometric analysis of ROS and the mitochondrial status by MitoTracker, CM-H2DCFDA, and MitoSOX staining. (e) Western blotting analysis of unsorted (W) and sorted P1 and P2 cells. Data shown are representative of at least two independent experiments. Data are shown as mean \pm s.e.m.

ferrochelatase (Fech). Haeme oxygenase-1 (HO-1) expression is induced by haeme to reduce the cellular haeme level. In contrast, CD71, the transferrin receptor, is required for iron uptake. The levels of haeme synthesis were not simply explained by expression levels of ALA synthase, CD71, or HO-1 (Supplementary Fig. 11). We accordingly investigated the processes of haeme synthesis affected by mROS by comparing the amounts of PpIX in the P1 and P2 cell populations. As shown in Fig. 4e, PpIX levels in P1 cells were higher than those in $\mathrm{P} 2$ cells, indicating that substrates and enzymes required to form the haeme precursor PpIX are not impaired but rather enhanced in the P1 cell population. We next assessed PpIX generation by the addition of ALA, whose synthesis is the rate-limiting step in haeme synthesis. PpIX levels were higher in P1 than in P2 cells, and this effect was counteracted by the treatment of P1 cells with MitoTEMPO (Fig. 4e-g). PpIX accumulation was simply explained by enhanced PpIX production or inhibition of the addition of ferrous ions to PpIX. The haeme level in P1 cells was low, and the antioxidant reversed the impairment of haeme synthesis (Fig. $4 \mathrm{a}-\mathrm{c}$ ). These data collectively suggested that mROS inhibited haeme synthesis by 

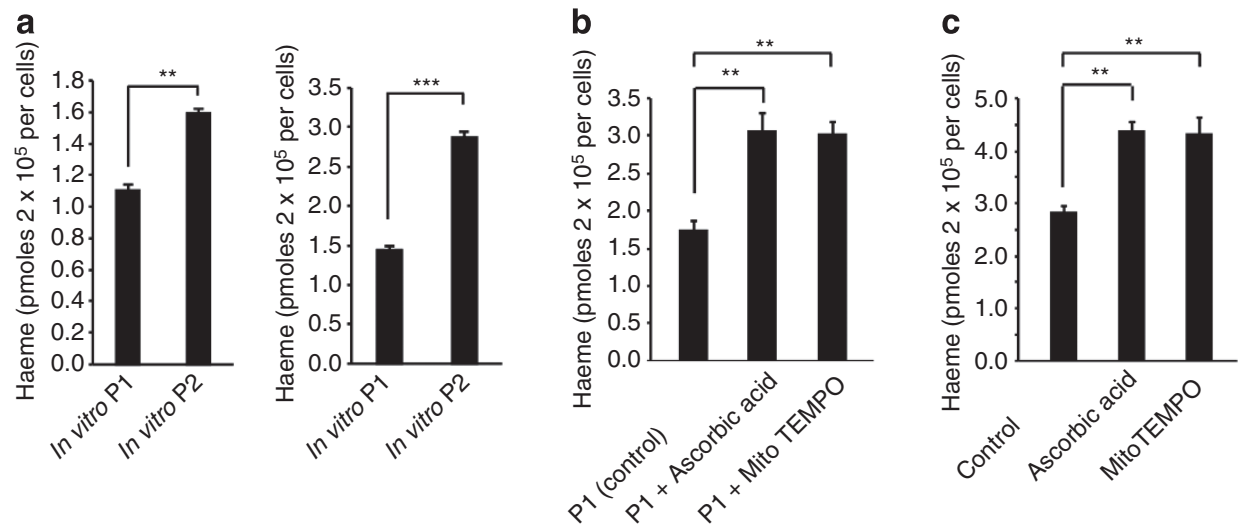

d
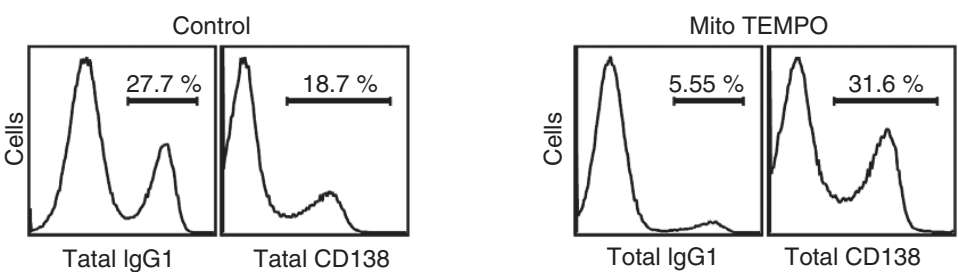

e

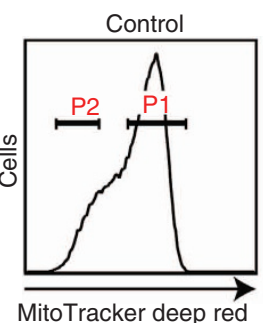

Control
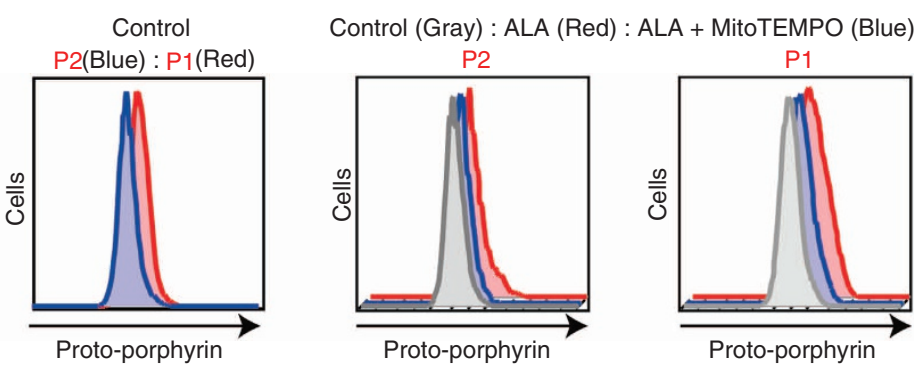

g

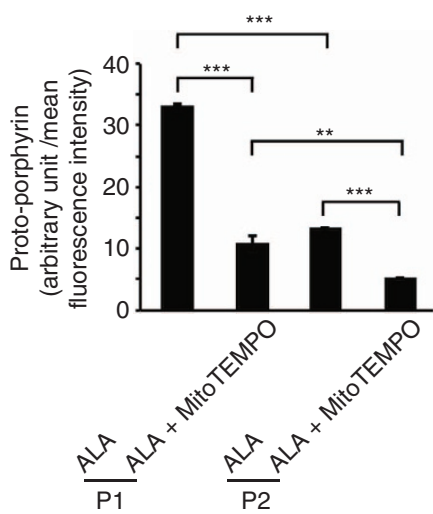

Figure 4 | Differential haeme synthesis in P1 and P2 cell populations. (a) Haeme levels in sorted P1 and P2 cells from in vitro-activated B cells (left) or GC $\mathrm{B}$ cells in an immunized spleen (right). (b) Haeme levels in sorted P1 cells treated with indicated antioxidants. (c) Haeme levels in in vitro-activated B cells treated with indicated antioxidants. (d) Flow cytometric analysis of the differentiation status monitored by CD138 and lgG1 expression after 4 days of culture with LPS + IL-4 in the presence or absence of MitoTEMPO. (e) Flow cytometric analysis of PplX fluorescence in P1 and P2 cells. In vitro-activated B cells treated with indicated reagents were assessed. (f) Mean fluorescence intensities (MFI) of PpIX as shown in (e) are plotted. (g) Increased MFI of PpIX after treatment with indicated reagents is plotted. Data shown are representative of three independent experiments. Data are shown as mean \pm s.e.m. ${ }^{\star \star} P<0.05,{ }^{\star \star \star} P<0.005$. (two-tailed Student's $t$-test).

interfering with the addition of ferrous ions to PpIX. However, the precise mechanisms by which mROS inhibited haeme synthesis await further investigation.

Haeme synthesis plays a key role in fate determination. To further confirm the role of de novo haeme synthesis in B-cell fate determination, we treated in vitro-activated B cells with ALA and $\mathrm{CoCl}_{2}$, a component of cobalt protoporphyrin (CoPP; not a substrate for HO-1 but an inhibitor of Bach function $)^{42}$. Consistently with the increased haeme level after the addition of ALA (Fig. 5a), PCD was promoted despite the high ROS level (Fig. 5b,c). This response strongly indicated that haeme synthesis itself, rather than ROS, plays a key role in PCD. This 
a

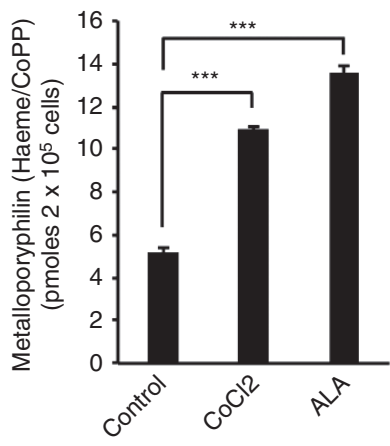

b

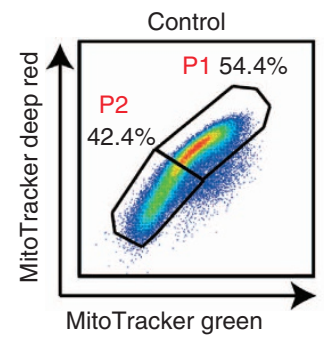

ALA

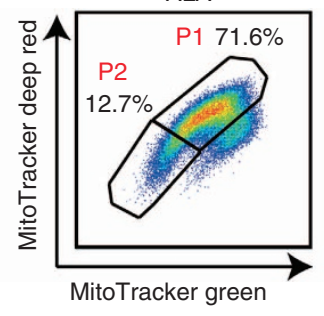

$\mathrm{CoCl} 2$
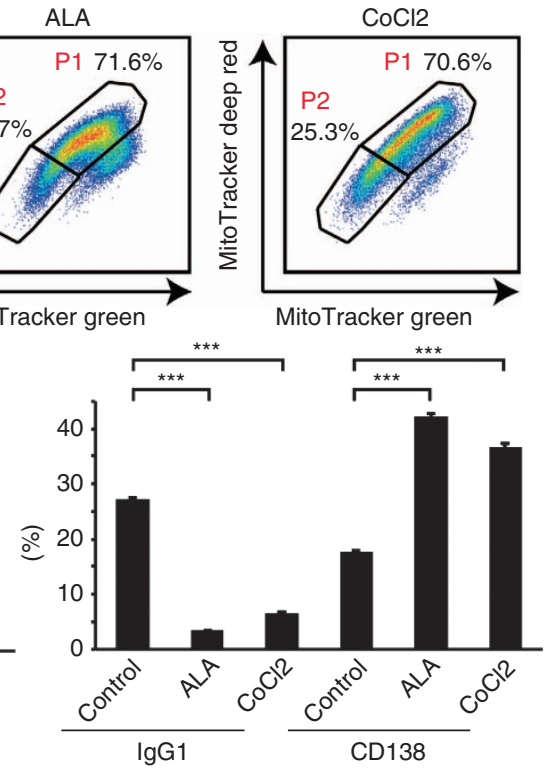

C
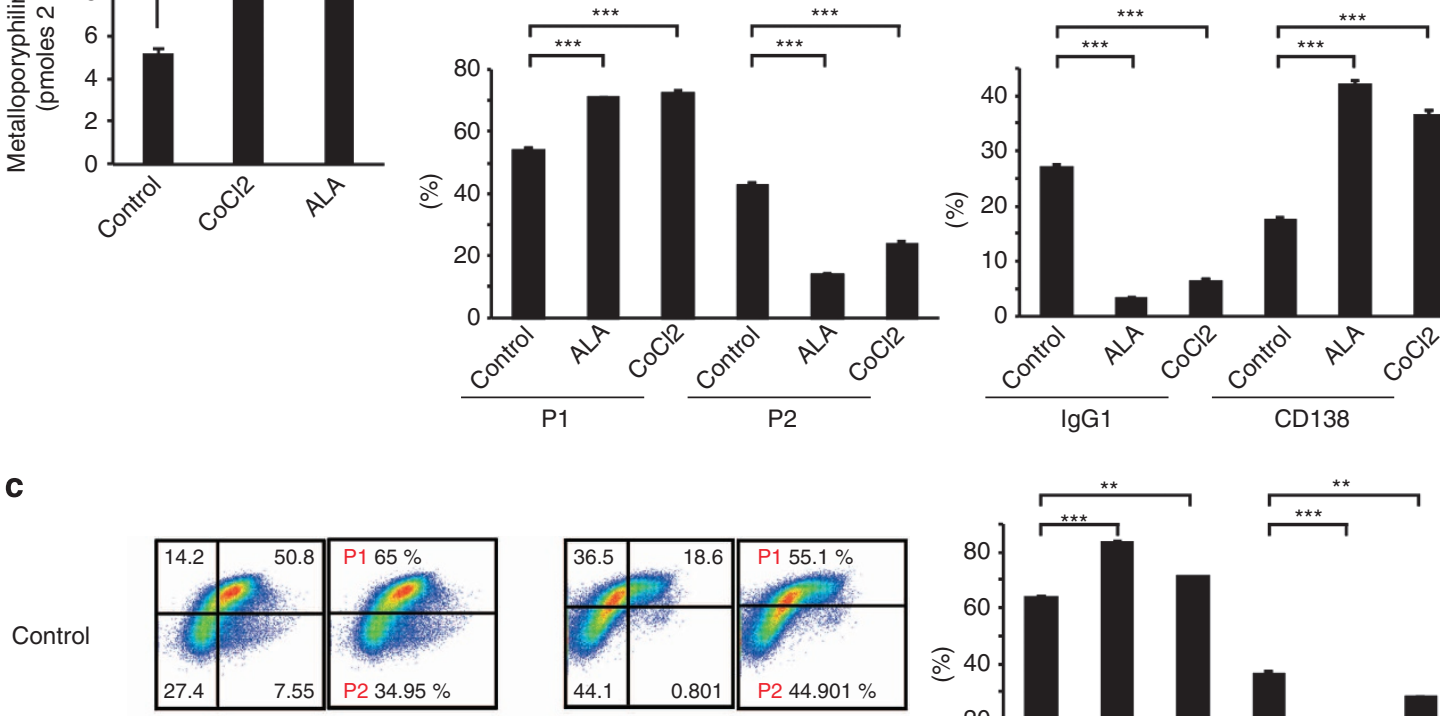

ALA
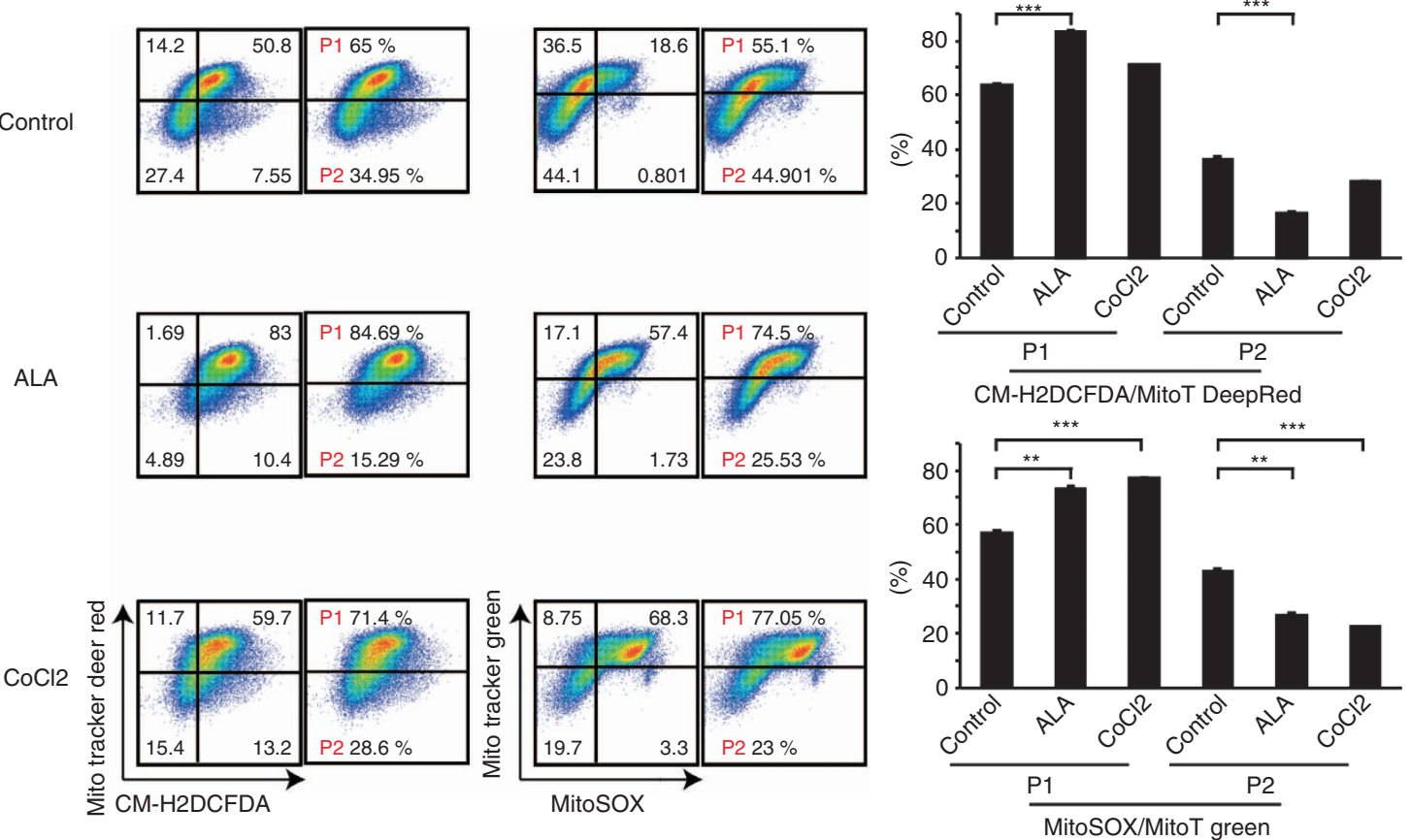

Figure 5 | Differential production of ROS instructs cell fates in activated B cells. (a) Metalloporphyrin levels of in vitro-activated B cells treated with indicated reagents. Metalloporphyrin in control or ALA-treated cells consisted of haeme, whereas that in $\mathrm{CoCl}_{2}$-treated cells consisted of haeme and CoPP. ${ }^{\star \star \star} P<0.005$ (two-tailed Student's t-test). (b) Flow cytometric analysis of mitochondrial status monitored by MitoTracker staining and differentiation status monitored by CD138 and IgG1 expression on day 4. ${ }^{\star \star \star} P<0.005$ (two-tailed Student's $t$-test). (c) Flow cytometric analysis of mitochondrial status and cellular ROS monitored by MitoTracker DeepRed and CM-H2DCFDA (left) staining or mitochondrial status and mROS monitored by MitoTracker Green and MitoSOX (right) staining. ${ }^{\star \star} P<0.05,{ }^{\star \star \star} P<0.005$. (two-tailed Student's $t$-test) FACS Data shown in $\mathbf{b}, \mathbf{c}$ are representative of three independent experiments. Data are shown as mean \pm s.e.m.

interpretation was further supported by the finding that PCD was enhanced after the treatment of activated $\mathrm{B}$ cells with $\mathrm{CoCl}_{2}$, which increases the amounts of metalloporphyrin, a molecule consisting of haeme and CoPP, and ROS level (Fig. 5a-c).

Bach2 inhibits P2 cell generation. We next examined the effects of Bach2 on mitochondrial status, because the mROShaeme relationship was based on modulating Bach2 function in
P1 and P2 cells. As shown in Fig. 6a, more P2 cells were generated from Bach $2^{-I-} \mathrm{B}$ cells than from wild-type cells, consistent with the observation that $\mathrm{P} 2$ cells contain more haeme than do P1 cells (Fig. 4a). These findings supported the notion that Bach2 function was suppressed in P2 cells and indicated that Bach2 was required for both P1 cells and CSR. Because Blimp1 expression is increased in Bach $2^{-/}-\mathrm{B}$ cells, we next investigated whether Blimp1 is required for $\mathrm{P} 2$ cell generation, using Blimp1-green fluorescent protein (GFP) knock-in mice. 

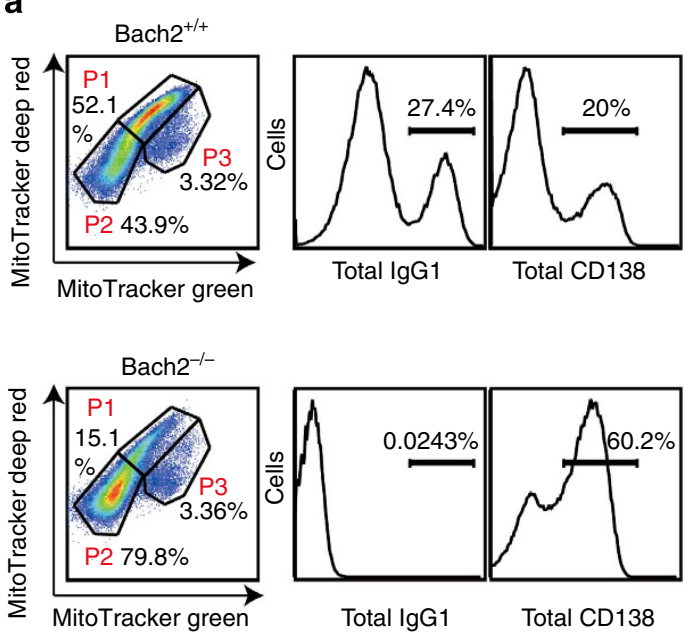

b $\quad B \operatorname{limp} 1^{+/ G}$

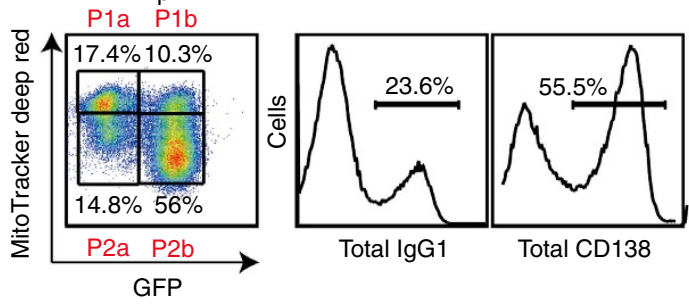

$B \operatorname{limp} 1^{\mathrm{G} / \mathrm{G}}$

P1a P1b
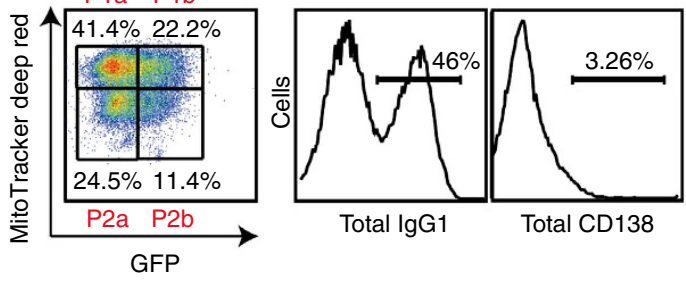

C

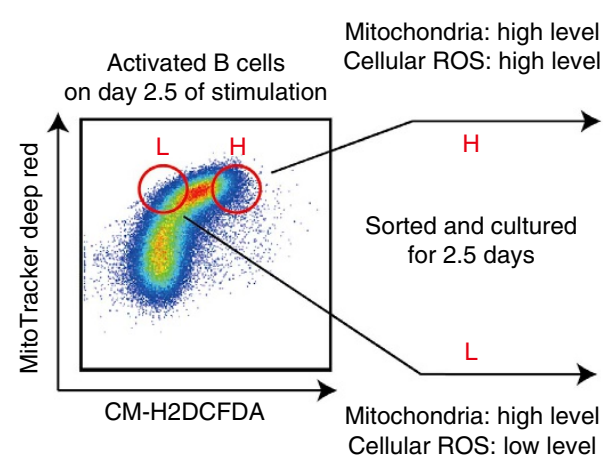

d
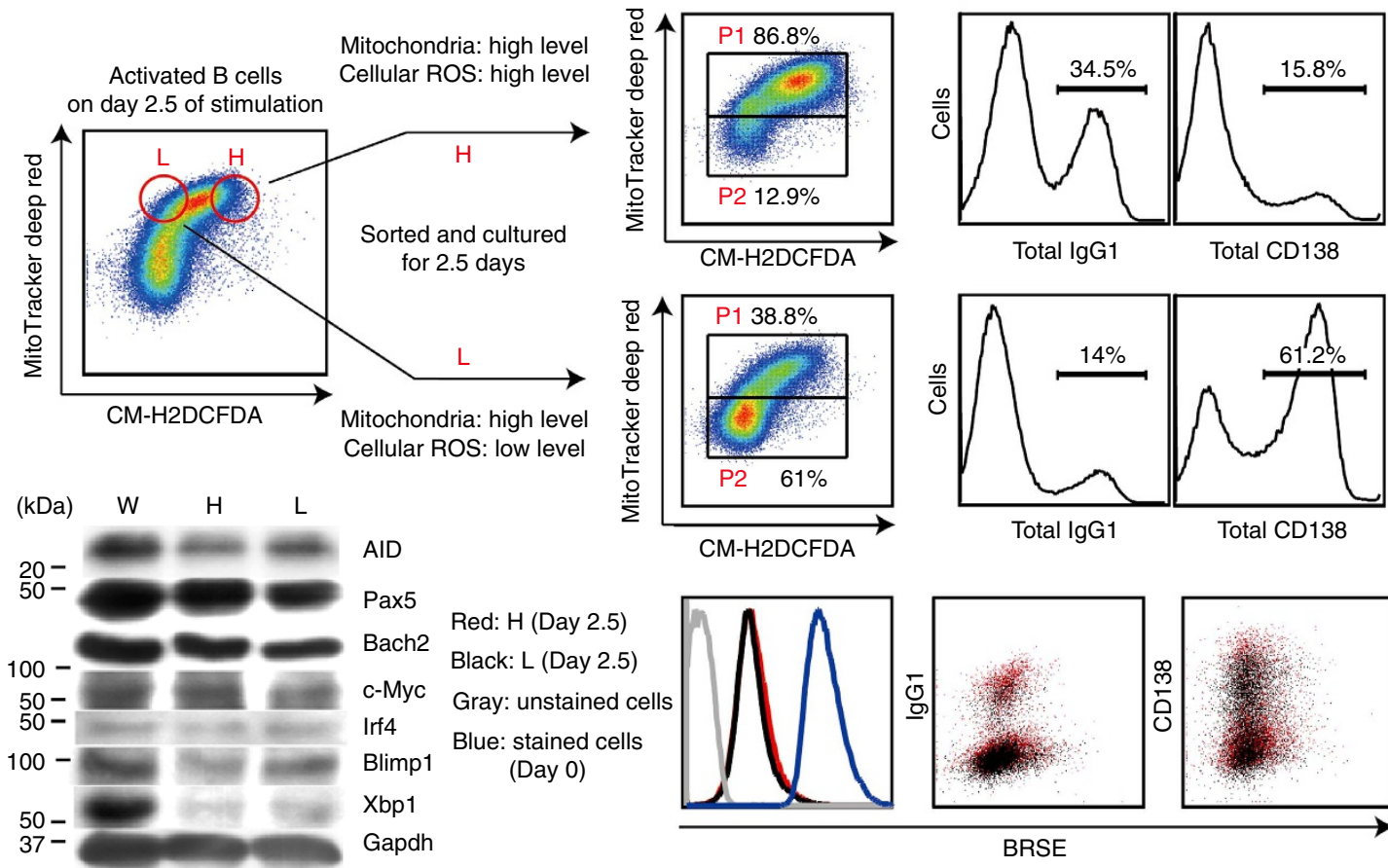

Total IgG1

Total CD138

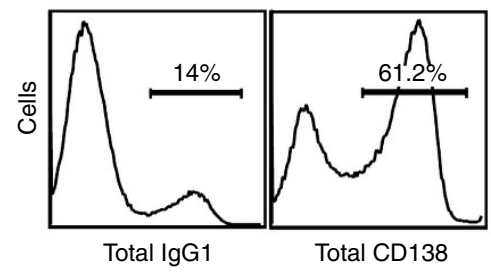

Red: H (Day 2.5)

Black: L (Day 2.5)

Gray: unstained cells

Blue: stained cells

(Day 0)
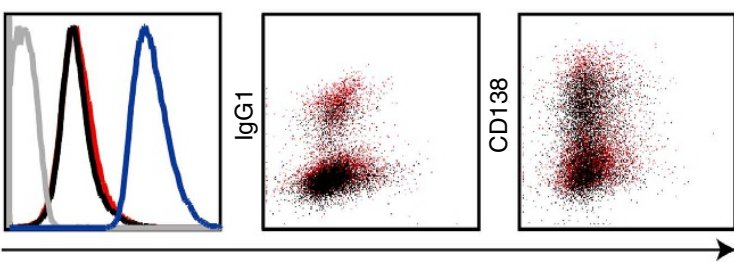

BRSE

Figure 6 | Differential ROS production provides instructive signals for fates of activated B cells. (a) Flow cytometric analysis of mitochondrial membrane potential and size monitored by MitoTracker staining (left) or differentiation of B cells monitored by CD138 and lgG1 expression (middle and right) in LPS + IL-4-stimulated Bach2 ${ }^{-/-}$or WT B cells. (b) Flow cytometric analysis of mitochondrial membrane potential monitored by MitoTracker staining and GFP expression (left) or differentiation of B cells monitored by CD138 and IgG1 expression (middle and right) in LPS + IL-4-stimulated Blimp $^{\mathrm{G} / \mathrm{G}}$ or Blimp $1^{\mathrm{G} /}+\mathrm{B}$ cells. (c) Diagrammatic representation of experimental protocol. Flow cytometric analysis of differentiation of sorted ROSlow $(\mathrm{L})$ and ROShigh $(H)$ cells (top and middle) and proliferation (bottom). (d) Western blotting analysis of unsorted (W) and sorted ROSlow $(\mathrm{L})$ and ROS high $(\mathrm{H})$ cells. Data shown are representative of at least two independent experiments.

In these mice, Blimp1 was replaced with GFP, so that GFP expression was under the control of endogenous Blimp1 regulatory elements, indicating that Blimp1-expressing cells was easily identified by GFP expression. Because committed cells to PCD, but not to CSR, express Blimp1, GFP ${ }^{+}$cells was PC-committed populations. P2 cells were generated from Blimp1-expressing cells $\left(\mathrm{GFP}^{+}\right.$cells in Blimp1 ${ }^{+/ G F P}$ B cells, Fig. 6b upper column) and could not be observed in Blimp1 ${ }^{\text {GFP/GFP }}$ B cells (Fig. $6 \mathrm{~b}$ lower column). These data indicated that P2 cells were generated in a manner depending on Blimp1 function and suggested that the mitochondrial status of P2 cells were a physiological property of differentiated plasma cells.
Differential ROS production provides instructive signals. As shown in Fig. 1c, commitment of activated B cells to CSR or PCD was predicted by their mitochondrial status. P1 cells, which contained higher mitochondrial mass and membrane potential, preferentially underwent CSR, whereas P2 cells, which contained lower mitochondrial mass and membrane potential, differentiated into plasma cells. Bach2 function is required for CSR, and reduced activity of Bach2 promotes PCD. However, the expression level of Bach2 differed little between P1 and P2 cells (Fig. 3e). Thus, the differential regulation of Bach2 activity is key in these cell fate-determination processes. Bach2 function was maintained by inhibition of haeme synthesis by mROS produced in P1 cells (Fig. 4a-g). In contrast, Bach2 function was inhibited in P2 cells, 
allowing the expression of Bach2 target genes such as Blimp1 (Fig. 3e). P2 cells appear to arise from plasma cell-committed populations (Figs $3 \mathrm{e}$ and $6 \mathrm{~b}$ ). To determine whether the same scenario is applicable in the initial step of cell fate determination, we evaluated the differentiation capacity of ROS ${ }^{\text {high }}$ and ROS low cells within P1 cells. Between ROS high and ROS ${ }^{\text {low }}$ cells derived from the P1 cell population, we found no differences in protein levels of transcription factors essential to CSR and PCD (Fig. 6d). These results suggested that cells at this stage are not committed to either differentiation pathway. When undifferentiated ROS ${ }^{\text {high }}$ and ROS ${ }^{\text {low }}$ cells were collected and cultured for an additional 2.5 days, ROS high cells underwent predominantly CSR, whereas ROS ${ }^{\text {low }}$ cells differentiated into plasma cells (Fig. 6c). In addition, the preferential appearance of P1 cells was observed in cultured
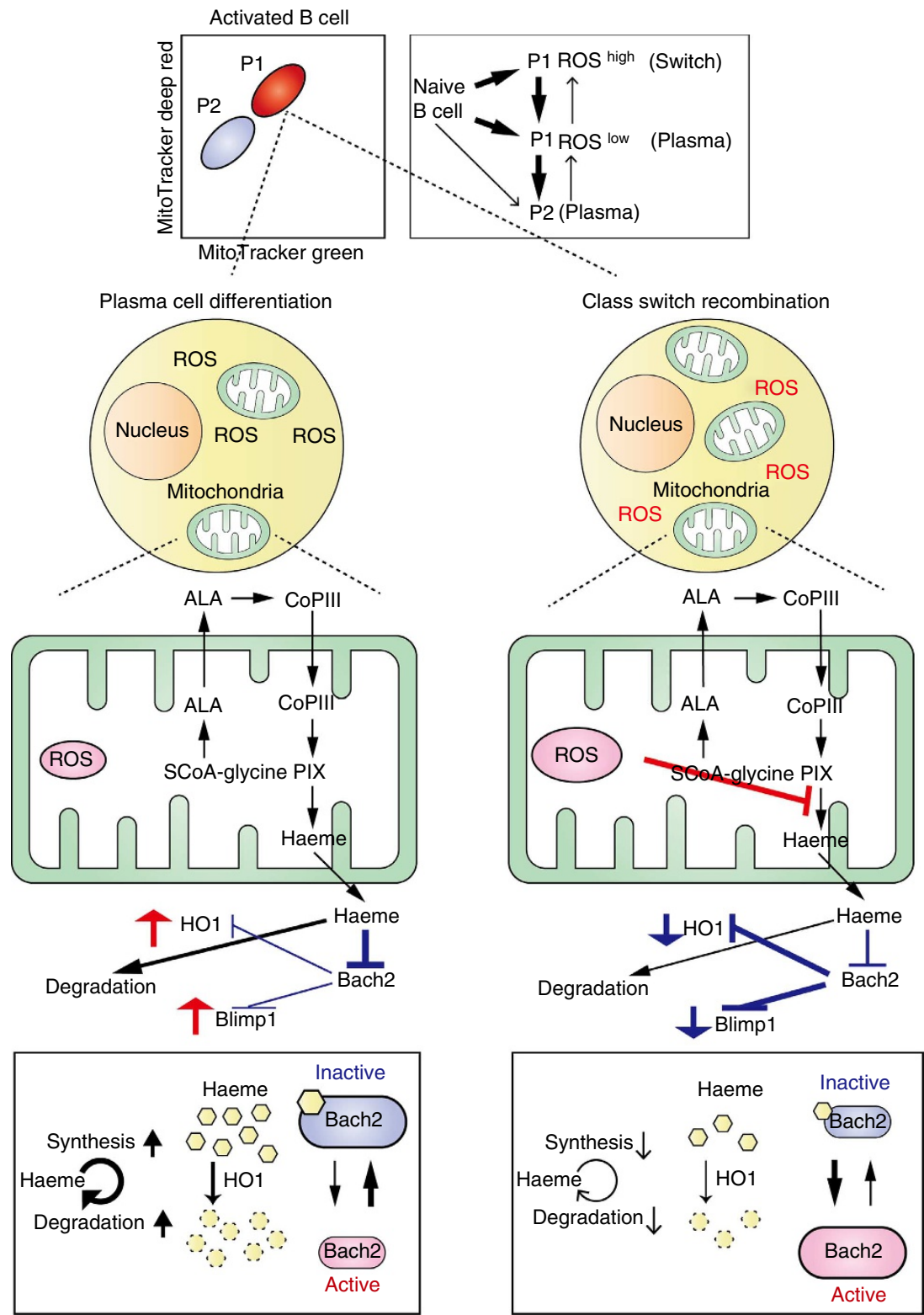

Figure 7 | Schematic representation of models of B-cell differentiation during normal course of immune reaction. On activation, B cells acquire mitochondrial mass and membrane potential (and are called P1 cells) and proliferate. Stochastic differences in mitochondrial activity within P1 cells generate diverse P1 cells having various ROS amounts (in this scheme, they are simply classified into two groups, P1 ROS ${ }^{\text {high }}$ and P1 ROSlow cells). In addition to the signalling function, excess amounts of ROS have another function of attenuating haeme synthesis. In general, newly generated haeme that cannot bind to haeme proteins is degraded immediately by HO-1. Haeme that binds to high- but not to low-affinity haeme-binding proteins (such as Bach2), are retained for a longer period. Thus, the effects of haeme on the regulation of low-affinity haeme-binding proteins are limited. Moreover, increasing amounts of free haeme induce HO-1 expression, which enhances the activity of free haeme clearance. Accordingly, the level of effective haeme concentration that regulates low-affinity haeme-binding proteins cannot be achieved by transient elevation of haeme contents. In P1 ROS ${ }^{\text {low }}$ cells, the upregulation of haeme turnover, which supports a continuous haeme supply, inhibits Bach2 function and promotes the PCD program (from P1 ROS low to P2 cells). Theoretically, initial changes might occur at any steps described in this scheme. For example, HO-1-dependent degradation of haeme provides an antioxidant, which can cause reduction of ROS level. The important steps in the respective immune reactions await identification. The majority of naïve B cells are also P2 cells, but almost all the cells show increased mitochondrial mass, membrane potential (Fig. 1a) and Bach2 protein expression after activation (not shown). Accordingly, P1 and P2 populations observed in naïve B cells may be different from P1 and P2 populations of activated B cells. 
ROS ${ }^{\text {high }}$ cells, but appearance of P2 cells was observed in cultured in ROS ${ }^{\text {low }}$ cells (Fig. 6c). Because CSR and PCD are influenced by cell proliferation, numbers of cell divisions were assessed by BRSE. As shown in Fig. 6c, there was little difference in the cell division numbers between these populations. These results indicated that change in mROS generation was the cause of the cell fate decision after B-cell activation. These findings together indicate that fate determination of activated B cells is dependent on ROS level, which negatively regulates haeme synthesis. Halflife of Bach2 protein was longer than that of Pax 5 protein, because significant amount of Bach2 protein was observed in 5 dayscultured B cells (Supplementary Fig. 12). These data indicated that quantitative regulation of haeme plays an important role in promoting PCD. In other words, PCD is a reversible state before loss of Bach2 protein. Our results show the mechanism of induction of differentiation in the lineage commitment process of B cells (Fig. 7). In general, commitment to specific lineages of haematopoietic cells is an integrated interpretation of instructive and stochastic signals ${ }^{14-17,43,44}$. Precise understanding of the mechanism of $\mathrm{B}$-cell fate determination requires further identification of the mechanisms by which levels of ROS and haeme synthesis are regulated by instructive signals and by which haeme functions as a signalling molecule.

\section{Discussion}

Fate determination of activated B cells toward CSR or PCD appears to be an intracellular stochastic process ${ }^{14,15}$. However, the underlying stochastic changes resulting in the initiation of either pathway are incompletely understood.

In this study, we identified mROS, which reflect individual mitochondrial status, as a signal for the initiation of fate determination of activated B cells. mROS promoted CSR and suppressed PCD by attenuating haeme synthesis because haeme inhibits Bach2 function ${ }^{36}$. The underlying mechanism of the inhibition of haeme synthesis is unknown. According to our combined experimental results, mROS inhibited the addition of ferrous ions to PpIX in mitochondria. There are at least three ways by which this inhibition may occur-mROS may inhibit haeme synthesis by inhibiting Fech activity; mROS may inhibit the reduction of ferric ion to ferrous ion, a substrate of Fech, in mitochondria; or there may be a defect in iron availability in mitochondria. In this regard, involvement of Fech in CSR and PCD was examined in vivo. As shown in Supplementary Fig. 13a, CSR was enhanced in mice treated with Fech inhibitor, but its $P$ value did not meet the criteria of statistical significance. Additionally, mitochondrial function in activated B-cell fate determination was investigated in vivo. Enhanced CSR and reduced PCD were observed in complexI/III-inhibitor-treated mice (Supplementary Fig. 13b).

$\mathrm{B}$ cells, having high affinity to B-cell receptors (BCR) and antigens, differentiate preferentially into plasma cells, indicating that antigen recognition strength is an instructive signal for PCD. PI3K and Akt activities affect activated B-cell fates ${ }^{38}$. The PI3KAkt pathway, downstream of BCR signalling, inhibits CSR by repressing AID gene expression via Foxo1 and Foxo3a phosphorylation, and promotes $\mathrm{PCD}$ by inducing Blimp1 expression. However, the mechanism by which PI3K-Akt induces Blimp1 is unknown. Another downstream signal of $\mathrm{BCR}$, Erk, is also required for PCD. In this case, Blimp1 expression is induced by Erk-dependent Elk1 phosphorylation ${ }^{45}$. Irf4 is required for both CSR and PCD ${ }^{12,13}$. The antigen-binding affinity of BCR regulates the magnitude of Irf4 expression and acts as a dose-dependent regulator of activated B-cell fates ${ }^{12,13}$. A weaker BCR signal induces a lower Irf4 level, resulting in CSR. In contrast, increased antigen affinity augments BCR signalling- mediated Irf4 expression and promotes PCD. Dose-dependent cell fate determination by Irf4 is achieved via discrimination between the affinities of various target sequences, as suggested previously ${ }^{7,46}$. Lower-affinity recognition sequences of Irf4 are occupied by Irf4 in differentiating plasma cells, which promote PCD by inducing the gene sets essential for PCD ${ }^{12}$. In T cells, Irf4 expression is also controlled by $\mathrm{T}$-cell receptor affinity ${ }^{47}$, indicating the presence of a similar regulatory network in $\mathrm{T}$ cells. Thus, a relationship between the amount of Irf4 and cell fate determination has been clearly demonstrated. However, the mechanisms by which the strength of BCR signalling is sensed and Irf4 expression is regulated are unknown.

Inhibition of the PI3K-Akt signal strongly promotes P1 cell generation, accompanied by reduced PCD and enhanced CSR. In other words, the PI3K-Akt signal, a downstream signal of BCR, promotes P2 cell generation, which in turn promotes PCD. An increased amount of $\mathrm{mROS}$ is produced in the $\mathrm{P} 1$ cell population, attenuating haeme synthesis. Bach2 function is maintained in the P1 cell population and suppressed in the P2 cell population because Bach2 is negatively regulated by haeme. In differentiating plasma cells, increasing amounts of haeme inhibit Bach2 function and in turn promote Blimp1 expression because Blimp1 is a direct target of Bach2 in B cells. Recent reports have shown that Irf4 is a direct target of Bach2 in macrophages ${ }^{48}$ or regulatory $\mathrm{T}$ cells ${ }^{49}$, indicating that reduced Bach2 function in the P2 cell population is responsible for the induction of Irf4 gene expression. Alternatively, the involvement of another transcriptional repressor also regulated by haeme is an attractive mechanism by which Irf4 expression may be induced in haeme-rich P2 cells. Mitf is a strong candidate as a transcriptional repressor of Irf4 in $\mathrm{B}$ cells ${ }^{50}$, and contains three Cys-Pro (CP) motifs involved in haeme binding. The involvement of Mitf in this haeme-decoding system awaits further elucidation.

In summary, we provide a model to explain how instructive and stochastic signals of activated B cells are integrated via changes in mitochondrial function that modulate mROS levels. Besides the function of mROS as signals for various cell fate determinations, mROS attenuate haeme synthesis, and the amount of haeme also plays a key role in cell fate determination of activated B cells by regulating the activities of haeme-binding transcription factors including Bach2. Accumulating evidence indicates that self-renewal and differentiation of HSCs are dependent on mROS, although the precise role of mROS in these processes is unclear. Thus, our findings provide insight into the fundamental roles of haeme synthesis, which is regulated by mitochondrial status, in cell fate determination processes. Identification of mROS as inhibitors of haeme synthesis has important implications for understanding not only B cells but also HSC biology, and may be applicable to the treatment of hematopoietic disorders.

\section{Methods}

Mice. This study received ethics approval from the Institutional Review Board of Kyoto University (reference number, Med Kyo13086). Bach2 ${ }^{-1-}$ (provided by Dr Igarashi, Tohoku University), Blimp1-GFP (provided by Dr Nutt, The Walter and Eliza Hall Institute of Medical Research) and wild-type mice on C57BL/6 genetic background were maintained in a specific pathogen-free mouse facility, and male mice were used at 8-12 weeks of age. Procedures involving animals and their care followed the guidelines for animal treatment of the Institute of Laboratory Animals, Kyoto University.

Cell preparation and in vitro culture. Naïve B cells were prepared by magnetic cell sorting using anti-CD43 microbeads (130-49-801; Miltenyi Biotec). Purified splenic B cells $\left(5 \times 10^{5}\right.$ cells $\left.\mathrm{ml}^{-1}\right)$ were cultured in RPMI 1640 medium (11875-093; Gibco) supplemented with $10 \%$ fetal bovine serum (FBS) and 2-mercaptoethanol ( $50 \mu \mathrm{M}$; Nacalai Tesque) in the presence of LPS $\left(40 \mu \mathrm{g} \mathrm{ml}^{-1}\right.$; L7136/015K4074/ 014K4112/086K4056 and L3755/102M4018V; Sigma) and IL-4 (20 ng ml ${ }^{-1}$; R\&D systems) or anti-mouse CD40 (HM40-3) antibody $\left(1 \mathrm{~g} \mathrm{ml}^{-1}\right.$; 553721 ; BD 
PharMingen) and IL-4 for 4 days. In some experiments, cells cultured for 2.5 days were sorted by flow cytometry using FACSAria (Becton Dickinson) and restimulated with LPS + IL-4 in the presence of the indicated reagents (Figs 1c and 6d).

CFSE or BRSE labelling. Purified splenic naïve B cells $\left(5 \times 10^{6}\right.$ cells $)$ were stained with $500 \mu \mathrm{l}$ of 5- $\mu \mathrm{M}$ CFSE solution (345-06441; DOJINDO) in a $\mathrm{CO}_{2}$ incubator at $37^{\circ} \mathrm{C}$ for $10 \mathrm{~min}$. Sorted cells were stained with $500 \mu \mathrm{l}$ of $5-\mu \mathrm{M}$ BRSE solution (D2219; Molecular Probes) in a $\mathrm{CO}_{2}$ incubator at $37^{\circ} \mathrm{C}$ for $10 \mathrm{~min}$.

Flow cytometric analysis and cell sorting. After cultured cells were washed with prewarmed no-glucose RPMI 1640 medium (11879020; Gibco) supplemented with $10 \%$ FBS (staining buffer), $1.5 \times 10^{6}$ cells were resuspended in $1 \mathrm{ml}$ of staining buffer containing MitoTracker Green (20 nM; M7514; Invitrogen), MitoTracker DeepRed (20 nM; M22426; Invitrogen), MitoSOX (5 $\mu \mathrm{M}$; M36008; Invitrogen), CM-H2DCFDA ( $5 \mu$ M; C6827; Invitrogen), TMRM ( $40 \mathrm{nM}$; 9105; Immunochemistry Technologies) or CellROX DeepRed ( $5 \mu \mathrm{M}$; C10422; Life Technologies) and incubated in a $\mathrm{CO}_{2}$ incubator at $37^{\circ} \mathrm{C}$ for $30-40 \mathrm{~min}$. Stained cells were washed with $1 \mathrm{ml}$ of prewarmed staining buffer and used for FACS (fluorescenceactivated cell sorting) analysis, cell sorting or further staining with antibodies. Normal rabbit serum (10\%) containing Fc Block (2.4G2; BD PharMingen) was used as blocking reagent. For immunized spleen cells, $5 \times 10^{6}$ cells were incubated with $1 \mathrm{ml}$ of staining buffer containing MitoTracker Green $(40 \mathrm{nM})$ and DeepRed $(40 \mathrm{nM})$, and acid wash treatment was applied to remove surface-bound immunoglobulin before antibody staining. MitoTracker-stained $5 \times 10^{6}$ spleen cells were suspended in $10 \mu \mathrm{l}$ of acid solution (equal volumes of cold $0.1 \mathrm{M}$ acetic acid, $\mathrm{pH} 4$ and solution containing $0.17 \mathrm{M} \mathrm{NaCl}$ and $0.01 \mathrm{M} \mathrm{KCl}$ mixed before use), incubated on ice for $1 \mathrm{~min}$ and neutralized by the addition of $1 \mathrm{ml}$ of cold Tris buffer $(0.03 \mathrm{M}$ Tris, $\mathrm{pH}$ 7.4, $0.8 \times$ PBS, $2 \%$ FCS). The following antibodies (1/300 diluted) were used for staining: biotin-labelled anti-mouse IgG1 (553441; BD PharMingen), phycoerythrin (PE)-conjugated anti-mouse CD138 (553714; BD PharMingen), Brilliant Violet 421-conjugated anti-mouse CD138 (562610; BD PharMingen), PE/ Cy7-conjugated anti-mouse B220 (552772; BD PharMingen), PE-conjugated antimouse FAS (554258; BD PharMingen), streptavidin-Brilliant Violet 421-conjugated (405226; Biolegend) and streptavidin-Brilliant Violet 570-conjugated (405227; Biolegend). For sorting immunized spleen cells, T-cell depletion was performed using IMAG and anti-Thy1.2 beads (551518; BD Biosciences) before staining with MitoTracker. Cytometric analysis and sorting of in vitro cultured and immunized cells was performed using FACSAria.

Immunization. For T-cell-dependent immune response, NP-chicken gamma globulin (100 $\mu \mathrm{g}$; NP-CGG; Biosearch Technologies) in complete Freund's adjuvant (Difco) was intraperitoneally injected. For T-cell-independent immune response, NP-Ficoll $(50 \mu \mathrm{g})$ in PBS was intraperitoneally injected.

Reagents used in vivo. During T-cell-independent immune reaction, $N$-methyl protoporphyrin $\left(16 \mathrm{~g} \mathrm{~kg}^{-1}\right.$ per day; MPP; Fech inhibitor; NMP576; Frontier Scientific) or $N$-benzylguanidine acetate $\left(10 \mathrm{~g} \mathrm{~kg}^{-1}\right.$ per day; NBA; complexes I, III inhibitor; SC-279892; SantaCruz) were intraperitoneally injected.

Reagents used in in vitro culture. The following reagents were added to in vitro culture from days 2.5 to 4 : methyl pyruvate ( $10 \mathrm{mM}$; 371173 ; Sigma), methyl malate ( $5 \mathrm{mM}$; 355-17971; Wako), etomoxir ( $90 \mu \mathrm{M}$; E1905; Sigma), rotenone (120 nM; R8875; Sigma), metformin (1 mM; 150959; SIGMA), oligomycin (1 nM; O4876; Sigma), 2-deoxy-D-glucose (200 $\mu \mathrm{M}$; 154-17-6; SIGMA), haemin ( $30 \mu \mathrm{M}$; H9039; Sigma), ascorbic acid (200 $\mu \mathrm{M}$; A7506; Sigma), MitoTEMPO (64 $\mu \mathrm{M}$; ALX430-150; Enzo Life Science) and TPP ( $64 \mu \mathrm{M}$; 309567; SIGMA); from days 1 to 4: LY294002 (3 $\mu \mathrm{M}$; 1667; Biovision), AZD5363 (5 $\mu \mathrm{M}$; S8019; Selleckchem) and 5ALA hydrochloride ( $450 \mu \mathrm{M} ; 3785$; Sigma); and from days 3 to 4 : cobalt (II) chloride (150 $\mu$ M; 232696; Sigma).

For OCR and ECAR assay, all the reagents were added at $36 \mathrm{~h}$ of culturing and incubated for $12 \mathrm{~h}$. Exposing duration of cells to reagents was therefore from 36 to $48 \mathrm{~h}$ of culturing.

Extracellular flux analysis. OCR and ECAR were measured in XF media (unbuffered RPMI 1640 containing $1 \%$ FBS, $11 \mathrm{mM}$ glucose, $2 \mathrm{mM}$ glutamine and $1 \mathrm{mM}$ pyruvate) under basal conditions and in response to $1 \mu \mathrm{M}$ oligomycin (O4876; Sigma), $1 \mu \mathrm{M}$ carbonyl cyanide 4-(trifluoromethoxy)phenylhydrazone and $1 \mu \mathrm{M}$ antimycin A (A0149; Sigma) on the XF96 extracellular flux analyzer (Seahorse Bioscience, Billerica, MA, USA). Basal OCR was calculated by subtraction of the residual rate after antimycin A treatment. After culture with LPS and IL-4 for 2.5 days, B cells were stained with MitoTracker Green and DeepRed and sorted using FACSAria. Sorted cells were cultured for $24 \mathrm{~h}$ under the same conditions to allow recovery from the stress of sorting. These cells were resuspended in XF media and were plated onto Seahorse cell plates $\left(1 \times 10^{5}\right.$ cells per well) coated with polyL-lysine hydrobromide (P2636; Sigma).

To assess the effects of reagents on ECAR and OCR, we prepared cells treated with various reagents under the conditions described in 'Reagents used in in vitro culture.' After culture with LPS and IL-4 for $48 \mathrm{~h}$, cells were resuspended in XF media and were plated onto Seahorse cell plates $\left(1 \times 10^{5}\right.$ cells per well) coated with poly-L-lysine hydrobromide (P2636; Sigma). OCR and ECAR were measured as described above.

Protein extraction and western blot analysis. Proteins were extracted with lysis buffer (Tri-HCl (pH 7.4), $150 \mathrm{mM} \mathrm{NaCl}, 1 \mathrm{mM}$ EDTA, $1 \%$ NP-40, $0.5 \%$ NaDOC, $0.1 \% \mathrm{SDS}, 1 \mathrm{mM} \mathrm{Na} \mathrm{VO}_{4}, 1 \mathrm{mM}$ phenylmethylsulphonyl fluoride, $1 \mu \mathrm{g} \mathrm{ml}{ }^{-1}$ pepstatin, $1 \mu \mathrm{g} \mathrm{ml}^{-1}$ aprotinine and $10 \mu \mathrm{g} \mathrm{ml}^{-1}$ leupeptine) for $20 \mathrm{~min}$ on ice, and protein amounts were quantified using Micro BCA Protein Assay Reagent (23235; Pierce Chemical Co.). Whole-cell lysates ( $40 \mu \mathrm{g}$ per lane) were resolved on SDSpolyacrylamide gels, electrotransferred onto polyvinylidene difluoride membranes and examined by immunoblot analysis. The primary antibodies used were antiPAX5 (610862; BD PharMingen), anti-BCL6 (\#4242; Cell Signaling), anti-c-MYC (NB600-302C; Novus Biologicals), anti-IRF4 (sc-6059; Santa Cruz), anti-BLIMP1 (NB600-235; Novus Biologicals), anti-XBP1 (sc-7160; Santa Cruz), anti-HO-1 (NBP1-97507; Novus Biologicals), anti-AID (provided by Dr Alt) and anti-GAPDH (\#2118; Cell Signaling). Full-sized scans of all western blots shown in Figs 3 and 6 and Supplementary Figs 11 and 12 are provided in Supplementary Fig. 14. Quantification of intensity was performed using an Image J (http://imagej.nih.gov/ij/).

Transmission electron microscopy. Sorted P1 and P2 cells (from B cells cultured for 3 days) were plated onto a Lab-Tek Chamber Slide (177445; Nunc), coated with poly-L-lysine hydrobromide and fixed with $4 \%$ paraformaldehyde $/ 2 \%$ glutaraldehyde. Sections were viewed with a Hitachi H-7650 transmission electron microscope.

Reverse transcription-PCR. Total RNAs were extracted from cultured B cells with TRIzol reagent (Gibco). Oligo (dT)-primed complementary DNAs were prepared with reverse transcriptase. Complementary DNAs were subjected to PCR reactions with the following primer pairs: Bach2 forward, 5'-CGCTGTCGAAAGAGGAAG CTGGAC-3'; Bach2 reverse, 5'-CCTGGATCTGCTCTGGACTCTGGA-3'; Pax5 forward, 5'-ATTGTCACAGGCCGAGACT-3'; Pax5 reverse, 5'-GCTGCAGGGC TGTAATAGT-3'; Gapdh forward, 5'-CCATCACCATCTTCCAGGA-3'; Gapdh reverse, $5^{\prime}$-CCTGCTTCACCACCTTCTT-3'.

Haeme quantification. Sorted or cultured B cells $\left(2 \times 10^{5}\right)$ were suspended in $500 \mu \mathrm{l}$ of saturated oxalic acid in water and heated at $121^{\circ} \mathrm{C}$ for $20 \mathrm{~min}$. Emission peaks at 608 and $662 \mathrm{~nm}$ using $400 \mathrm{~nm}$ excitation were measured (Hitachi MPF-4).

Intracellular measurement of PpIX fluorescence. Intracellular PpIX fluorescence was evaluated by FACSAria (excitation $405 \mathrm{~nm}$; emission, 625/20 nm filter).

Intracellular ATP quantification. ATP was measured using an ATP determination kit (A22066; Invitrogen) according to the manufacturer's instructions.

Statistical analysis. OCR, ECAR, ATP, protoporphyrin, haeme and metalloporphyrin amounts were compared by Student's $t$-test. All reported $P$ values were twotailed and $P$ values $<0.05$ were considered to indicate significance.

\section{References}

1. Honjo, T., Kinoshita, K. \& Muramatsu, M. Molecular mechanism of class switch recombination: linkage with somatic hypermutation. Annu. Rev. Immunol. 20, 165-196 (2002).

2. Muto, A. et al. The transcriptional programme of antibody class switching involves the repressor Bach2. Nature 429, 566-571 (2004).

3. Ramiro, A. R. \& Nussenzweig, M. C. Immunology: aid for AID. Nature 430, 980-981 (2004).

4. Chaudhuri, J. \& Alt, F. W. Class-switch recombination: interplay of transcription, DNA deamination and DNA repair. Nat. Rev. Immunol. 4, 541-552 (2004).

5. Sugai, M., Gonda, H., Nambu, Y., Yokota, Y. \& Shimizu, A. Accessibility control of recombination at immunoglobulin locus. Curr. Immunol. Rev. 1, 69-79 (2005).

6. Nambu, Y. et al. Transcription-coupled events associating with immunoglobulin switch region chromatin. Science 302, 2137-2140 (2003).

7. Gonda, H. et al. The balance between Pax 5 and Id 2 activities is the key to AID gene expression. J. Exp. Med. 198, 1427-1437 (2003).

8. Shaffer, A. L. et al. XBP1, downstream of Blimp-1, expands the secretory apparatus and other organelles, and increases protein synthesis in plasma cell differentiation. Immunity 21, 81-93 (2004)

9. Nutt, S. L., Fairfax, K. A. \& Kallies, A. BLIMP1 guides the fate of effector B and T cells. Nat. Rev. Immunol. 7, 923-927 (2007).

10. Shapiro-Shelef, M. et al. Blimp-1 is required for the formation of immunoglobulin secreting plasma cells and pre-plasma memory B cells. Immunity 19, 607-620 (2003). 
11. Klein, U. et al. Transcription factor IRF4 controls plasma cell differentiation and class-switch recombination. Nat. Immunol. 7, 773-782 (2006).

12. Ochiai, K. et al. Transcriptional regulation of germinal center $B$ and plasma cell fates by dynamical control of IRF4. Immunity 38, 918-929 (2013).

13. Sciammas, R. et al. Graded expression of interferon regulatory factor-4 coordinates isotype switching with plasma cell differentiation. Immunity 25, 225-236 (2006).

14. Tarlinton, D. B-cell differentiation: instructive one day, stochastic the next Curr. Biol. 22, R235-R237 (2012).

15. Duffy, K. R. et al. Activation-induced B cell fates are selected by intracellular stochastic competition. Science 335, 338-341 (2012).

16. Barnett, B. E. et al. Asymmetric B cell division in the germinal center reaction. Science 335, 342-344 (2012).

17. Thaunat, O. et al. Asymmetric segregation of polarized antigen on B cell division shapes presentation capacity. Science 335, 475-479 (2012).

18. Horcher, M., Souabni, A. \& Busslinger, M. Pax5/BSAP maintains the identity of B cells in late B lymphopoiesis. Immunity 14, 779-790 (2001).

19. Delogu, A. et al. Gene repression by Pax 5 in B cells is essential for blood cell homeostasis and is reversed in plasma cells. Immunity 24, 269-281 (2006).

20. Lin, K. I., Angelin-Duclos, C., Kuo, T. C. \& Calame, K. Blimp-1-dependent repression of Pax-5 is required for differentiation of $\mathrm{B}$ cells to immunoglobulin M-secreting plasma cells. Mol. Cell. Biol. 22, 4771-4780 (2002).

21. Ochiai, K. et al. Plasmacytic transcription factor Blimp-1 is repressed by Bach2 in B cells. J. Biol. Chem. 281, 38226-38234 (2006).

22. Ochiai, K., Muto, A., Tanaka, H., Takahashi, S. \& Igarashi, K. Regulation of the plasma cell transcription factor Blimp-1 gene by Bach2 and Bcl6. Int. Immunol. 20, 453-460 (2008).

23. Reimold, A. M. et al. Plasma cell differentiation requires the transcription factor XBP-1. Nature 412, 300-307 (2001).

24. Shaffer, A. L. et al. Blimp-1 orchestrates plasma cell differentiation by extinguishing the mature B cell gene expression program. Immunity 17, 51-62 (2002).

25. Shlomchik, M. J. \& Weisel, F. Germinal center selection and the development of memory B and plasma cells. Immunol. Rev. 247, 52-63 (2012).

26. Vinuesa, C. G., Linterman, M. A., Goodnow, C. C. \& Randall, K. L. T cells and follicular dendritic cells in germinal center B-cell formation and selection. Immunol. Rev. 237, 72-89 (2010).

27. Victora, G. D. et al. Germinal center dynamics revealed by multiphoton microscopy with a photoactivatable fluorescent reporter. Cell 143, 592-605 (2010).

28. Calado, D. P. et al. The cell-cycle regulator c-Myc is essential for the formation and maintenance of germinal centers. Nat. Immunol. 13, 1092-1100 (2012).

29. Dominguez-Sola, D. et al. The proto-oncogene MYC is required for selection in the germinal center and cyclic reentry. Nat. Immunol. 13, 1083-1091 (2012).

30. Pearce, E. L., Poffenberger, M. C., Chang, C. H. \& Jones, R. G. Fueling immunity: insights into metabolism and lymphocyte function. Science 342, 1242454 (2013)

31. Tannahill, G. M. et al. Succinate is an inflammatory signal that induces IL-1beta through HIF-1alpha. Nature 496, 238-242 (2013).

32. Chang, C. H. et al. Posttranscriptional control of $\mathrm{T}$ cell effector function by aerobic glycolysis. Cell 153, 1239-1251 (2013).

33. Pearce, E. L. et al. Enhancing CD8 T-cell memory by modulating fatty acid metabolism. Nature 460, 103-107 (2009).

34. Takubo, K. et al. Regulation of glycolysis by Pdk functions as a metabolic checkpoint for cell cycle quiescence in hematopoietic stem cells. Cell Stem Cell 12, 49-61 (2013).

35. Sena, L. A. \& Chandel, N. S. Physiological roles of mitochondrial reactive oxygen species. Mol. Cell 48, 158-167 (2012).

36. Watanabe-Matsui, M. et al. Heme regulates B-cell differentiation, antibody class switch, and heme oxygenase-1 expression in B cells as a ligand of Bach2. Blood 117, 5438-5448 (2011).

37. Zhou, R., Yazdi, A. S., Menu, P. \& Tschopp, J. A role for mitochondria in NLRP3 inflammasome activation. Nature 469, 221-225 (2011)

38. Omori, S. A. et al. Regulation of class-switch recombination and plasma cell differentiation by phosphatidylinositol 3-kinase signaling. Immunity 25, 545-557 (2006)

39. Tormos, K. V. et al. Mitochondrial complex III ROS regulate adipocyte differentiation. Cell Metab. 14, 537-544 (2011).

40. Sena, L. A. et al. Mitochondria are required for antigen-specific T cell activation through reactive oxygen species signaling. Immunity 38, 225-236 (2013).
41. Muto, A. et al. Bach2 represses plasma cell gene regulatory network in B cells to promote antibody class switch. EMBO J. 29, 4048-4061 (2010).

42. Shan, Y., Lambrecht, R. W., Donohue, S. E. \& Bonkovsky, H. L. Role of Bach1 and Nrf2 in up-regulation of the heme oxygenase-1 gene by cobalt protoporphyrin. FASEB J. 20, 2651-2653 (2006).

43. Broxmeyer, H. E. \& Mantel, C. A ROSy future for metabolic regulation of HSC division. Nat. Med. 18, 1334-1336 (2012).

44. Kueh, H. Y., Champhekhar, A., Nutt, S. L., Elowitz, M. B. \& Rothenberg, E. V. Positive feedback between PU.1 and the cell cycle controls myeloid differentiation. Science 341, 670-673 (2013).

45. Yasuda, T. et al. ERKs induce expression of the transcriptional repressor Blimp1 and subsequent plasma cell differentiation. Sci. Signal. 4, ra25 (2011).

46. DeKoter, R. P. \& Singh, H. Regulation of B lymphocyte and macrophage development by graded expression of PU.1. Science 288, 1439-1441 (2000).

47. Man, K. et al. The transcription factor IRF4 is essential for TCR affinitymediated metabolic programming and clonal expansion of T cells. Nat. Immunol. 14, 1155-1165 (2013).

48. Nakamura, A. et al. Transcription repressor Bach2 is required for pulmonary surfactant homeostasis and alveolar macrophage function. J. Exp. Med. 210, 2191-2204 (2013).

49. Roychoudhuri, R. et al. $\mathrm{BACH} 2$ represses effector programs to stabilize T(reg)mediated immune homeostasis. Nature 498, 506-510 (2013).

50. Lin, L., Gerth, A. J. \& Peng, S. L. Active inhibition of plasma cell development in resting B cells by microphthalmia-associated transcription factor. J. Exp. Med. 200, 115-122 (2004).

\section{Acknowledgements}

We thank Drs F. Alt (Harvard University), U. Basu (Harvard University) and J. Chaudhuri (Memorial Sloan-Kettering Cancer Center) for providing AID antibody; Drs K. Iwai (Kyoto University), Y. Sasaki (Kyoto University), H. Arakawa (National Cancer Center) and Y. Yokota (Fukui University) for discussion of the findings; Drs K. Ochiai (Tohoku University) and Y. Yokota (Fukui University) for critical reading of the manuscript; Dr M. Minami (Kyoto University) for comments on mitochondrial assay; Ms K. Furuta and Mr H. Koda (Kyoto University) for technical assistance in electron microscopy; Drs Okuno (Kyoto University) and Fukuura (PRIMETECH Corp) for the XF96 assay; Dr Takatani (Kyoto University) for comments on this study; and Mr K. Yoshida (Kyoto University) for supporting experiments. We thank Enago (www.enago.jp) for English language review. This work was supported in part by grants-in-aid for Science Research on Priority Areas from the Ministry of Education, Culture, Sports, Science and Technology of Japan. S.L.N. was supported by an Australian Research Council Future Fellowship and Victorian State Government Operational Infrastructure Support and Australian Government NHMRC IRIIS

\section{Author contributions}

K.-J.J., H.M., A.M. and M.S. performed the experiments; A.M., K.A., T.H., Y.N., K.T., K.I. and S.T. assisted with the experiments; K.I. and S.L.N. provided the mice; M.S. designed and conceptualized the study; Y.N. performed the statistical analysis; Y.N., K.A., S.T., S.L.N. and K.I. made comments; A.S., K.-J.J., S.T., K.I. and M.S. interpreted the data; and M.S. wrote the manuscript.

\section{Additional information}

Supplementary Information accompanies this paper at http://www.nature.com/ naturecommunication

Competing financial interests: The authors declare no competing financial interests.

Reprints and permission information is available online at http://npg.nature.com/ reprintsandpermissions/

How to cite this article: Jang, K.-J. et al. Mitochondrial function provides instructive signals for activation-induced B-cell fates. Nat. Commun. 6:6750 doi: $10.1038 /$ ncomms7750 (2015)

This work is licensed under a Creative Commons Attribution 4.0 International License. The images or other third party material in this article are included in the article's Creative Commons license, unless indicated otherwise in the credit line; if the material is not included under the Creative Commons license, users will need to obtain permission from the license holder to reproduce the material. To view a copy of this license, visit http://creativecommons.org/licenses/by/4.0/ 